Article

\title{
The Transient Receptor Potential Melastatin 7 (TRPM7) Inhibitors Suppress Seizure-Induced Neuron Death by Inhibiting Zinc Neurotoxicity
}

\author{
Jeong Hyun Jeong ${ }^{1}$, Song Hee Lee ${ }^{1}$, A Ra Kho ${ }^{1}$, Dae Ki Hong ${ }^{1}$, Dong Hyeon Kang ${ }^{1}$, \\ Beom Seok Kang ${ }^{1}$, Min Kyu Park ${ }^{1}$, Bo Young Choi ${ }^{1, *}$, Hui Chul Choi ${ }^{2, *}$, Man-Sup Lim ${ }^{3, *}$ \\ and Sang Won Suh ${ }^{1, *}$ \\ 1 Department of Physiology, Hallym University, College of Medicine, Chuncheon 24252, Korea; \\ jd1422@hanmail.net (J.H.J.); sshlee@hallym.ac.kr (S.H.L.); arakho136@naver.com (A.R.K.); \\ zxnm01220@gmail.com (D.K.H.); ehdgus6312@gmail.com (D.H.K.); ttiger1993@gmail.com (B.S.K.); \\ bagmingyu50@gmail.com (M.K.P.) \\ 2 Department of Neurology, Hallym University, College of Medicine, Chuncheon 24252, Korea \\ 3 Department of Medical Education, Hallym University, College of Medicine, Chuncheon 24252, Korea \\ * Correspondence: bychoi@hallym.ac.kr (B.Y.C.); dohchi@naver.com (H.C.C.); ellemes@hallym.ac.kr (M.-S.L.); \\ swsuh@hallym.ac.kr (S.W.S.); Tel.: +82-10-8573-6364 (S.W.S.)
}

Received: 14 September 2020; Accepted: 23 October 2020; Published: 24 October 2020

\begin{abstract}
Transient receptor potential melastatin 7 (TRPM7) is an ion channel that mediates monovalent cations out of cells, as well as the entry of divalent cations, such as zinc, magnesium, and calcium, into the cell. It has been reported that inhibitors of TRPM7 are neuroprotective in various neurological diseases. Previous studies in our lab suggested that seizure-induced neuronal death may be caused by the excessive release of vesicular zinc and the subsequent accumulation of zinc in the neurons. However, no studies have evaluated the effects of carvacrol and 2-aminoethoxydiphenyl borate (2-APB), both inhibitors of TRPM7, on the accumulation of intracellular zinc in dying neurons following seizure. Here, we investigated the therapeutic efficacy of carvacrol and 2-APB against pilocarpine-induced seizure. Carvacrol $(50 \mathrm{mg} / \mathrm{kg})$ was injected once per day for 3 or 7 days after seizure. 2-APB ( $2 \mathrm{mg} / \mathrm{kg})$ was also injected once per day for 3 days after seizure. We found that inhibitors of TRPM7 reduced seizure-induced TRPM7 overexpression, intracellular zinc accumulation, and reactive oxygen species production. Moreover, there was a suppression of oxidative stress, glial activation, and the blood-brain barrier breakdown. In addition, inhibitors of TRPM7 remarkably decreased apoptotic neuron death following seizure. Taken together, the present study demonstrates that TRPM7-mediated zinc translocation is involved in neuron death after seizure. The present study suggests that inhibitors of TRPM7 may have high therapeutic potential to reduce seizure-induced neuron death.
\end{abstract}

Keywords: epilepsy; pilocarpine; carvacrol; 2-APB; transient receptor potential melastatin 7; zinc; neuron death; reactive oxygen species

\section{Introduction}

Epilepsy is a neurological disorder characterized by unpredictable seizure behavior [1-3]. Epileptic seizures are symptoms of abnormally excessive or synchronous neuronal activity in the brain [2]. The most common form of epilepsy, which does not occur congenitally, is temporal lobe epilepsy (TLE), which is one of the most common and deadly neurological disorders and one of the leading causes of cognitive impairment [4]. Epileptic seizures can also be caused by various types of brain damage, such as hypoxia, head trauma, or multiple recessive seizures $[5,6]$. Seizure activity may 
be disseminated through the brain's endogenous electrical fields. The anticipated mechanisms that may diffuse the spread and recruitment of neurons produce an increase in $\mathrm{K}^{+}$concentration outside of the cell and an increase in $\mathrm{Ca}^{2+}$ in the presynaptic terminals [7]. These mechanisms may blunt hyperpolarization and depolarize proximate neurons, as well as increase neurotransmitter release. Another mechanism of epilepsy that occurs after brain injury is an imbalance between neuronal excitation and inhibition, such as the up-regulation of excitatory circuits or the down-regulation of inhibitory circuits $[8,9]$. Thus, persistent and uncontrolled epilepsy can cause permanent brain damage and result in a cognitive decline [3]. In particular, epileptic seizures have a severe deteriorating effect on the function of the hippocampus, causing neuronal damage and gradually accumulating harmful cellular and metabolic changes $[10,11]$. In addition, epilepsy has been shown to increase spontaneous seizures by altering the development of the hippocampus and synapse formation [12]. Nevertheless, many survivors of severe epileptic seizures still show neuronal damage and cognitive impairment, while the rate of cure is significantly lower [13,14].

Zinc is the second most abundant transition metal after iron in the central nervous system $[15,16]$. Zinc modulates various physiological functions, such as cell division, proliferation, migration, development, and DNA synthesis $[17,18]$. Zinc deficiency can degrade the performance of short-term memory operations. Conversely, too much zinc is known to drive increased neuronal death after brain injury. Therefore, it is important to maintain the homeostasis of zinc under physiological conditions [19-21]. Most zinc exists in association with zinc-binding protein. However, in cases of neurological damage, excessive zinc is released into the extracellular space from the presynaptic terminals of neurons and then translocates into the intracellular space of postsynaptic neurons $[20,22]$. This leads to increased levels of intracellular free zinc, which can produce neurotoxicity [21]. Previous studies have consistently demonstrated that the influx of excess zinc into neurons following neurological disease, such as epilepsy [19,23-26], ischemia [27], trauma [28], and hypoglycemia [29,30], induces neuronal death.

The transient receptor potential melastatin 7 (TRPM7), belonging to the subfamily of the TRP channel, is an ion channel that regulates monovalent cations out of cells and the entry of divalent cations, such as zinc, magnesium, and calcium, into the cell [31]. In addition, TRPM7 also mediates the movement of divalent cations out of cells, as well as the entry of monovalent cations. In such cases, the extracellular divalent cation concentration is much higher than the intracellular concentration, but $\mathrm{K}^{+}$can escape via TRPM7 [32]. TRPM7 is the only known zinc permeable channel among the TRP family of ion channels $[33,34]$. In addition, the TRPM7 channel offers zinc permeability that is four-fold higher than calcium [33]. Previous studies have shown that the knockdown of TRPM7 with small interfering RNA and nonspecific TRPM7 inhibitors, such as $\mathrm{Gd}^{3+}$ or 2-aminoethoxydiphenyl borate (2-APB), decrease zinc neurotoxicity in cultured mouse cortical neurons [35].

Carvacrol, which is an essential oil extracted from Origanum vulgare (oregano), is well known to effectively act as an inhibitor of TRPM7 [36,37]. Carvacrol, a monoterpenoid phenol, is not a specific inhibitor of TRPM7 and may have other effects dependent on binding to other TRPC channels [38,39]. Carvacrol treatment reduced recurrent status epilepticus (SE)-induced cell death in cornu Ammonis 1 (CA1) and hilus [40], possibly through its action on TRPM7 channels. A recent study demonstrated that carvacrol inhibits lipopolysaccharide (LPS)-induced pro-inflammatory activation in RAW 264.7 macrophages via the ERK1/2 and NF-kB pathways [41]. In addition, there are recent reports that carvacrol has a neuroprotective effect by reducing TRPM7 overexpression and caspase 3 activity in a hemiparkinsonian model [42]. Moreover, there are recent reports that essential extracts such as carvacrol have anticonvulsant effects $[43,44]$. In addition, it has been reported that carvacrol has a neuroprotective effect on central nervous system (CNS) diseases, such as ischemia, and traumatic brain injury (TBI) [45-48]. To further confirm the conclusion, we determined whether 2-aminoethoxydiphenyl borate (2-APB), another non-specific inhibitor of TRPM7, promoted neuroprotective effects after seizure with a pilocarpine-induced SE model [49]. 2-APB is a membrane permeable lipophilic compound that acts as a blocker of the intracellular inositol 1,4,5-trisphosphate receptor $\left(\mathrm{IP}_{3} \mathrm{R}\right)[50,51]$. Although 2-APB 
is not specific, it is also known to act as an inhibitor of TRPM7 [52,53]. A recent study demonstrated that 2-APB has neuroprotective effects after ischemia by inhibiting the TRPM7 channel [54].

Therefore, the present study tested our hypothesis that TRPM7 channel inhibition may prevent zinc translocation and subsequent neuronal death after epilepsy. To evaluate our hypothesis, we used two different TRPM7 inhibitors, carvacrol and 2-APB, using lithium-pilocarpine-induced status epilepticus (SE) as a model for TLE.

\section{Results}

\subsection{Carvacrol Reduces Seizure-Induced TRPM7 Overexpression, Zinc Accumulation, and Neuronal Death}

To test whether blocking the TRPM7 channel by carvacrol could reduce the accumulation of intracellular free zinc and thereby rescue neurons from delayed neuronal death, we used a lithium-pilocarpine-induced SE model. Carvacrol was injected intraperitoneally once per day at a dose of $50 \mathrm{mg} / \mathrm{kg}$ for the entire experimental period following pilocarpine-induced SE. The brains were harvested at the designated time after SE for histological evaluation (Figure 1A). We first determined seizure behaviors according to the Racine scale [55] and used only SE-induced rats in this experiment (Figure 1B). Furthermore, we assessed weight change as a possible side effect after application of carvacrol. However, carvacrol treatment did not result in significant side effects in terms of weight loss (Figure 1C,D).

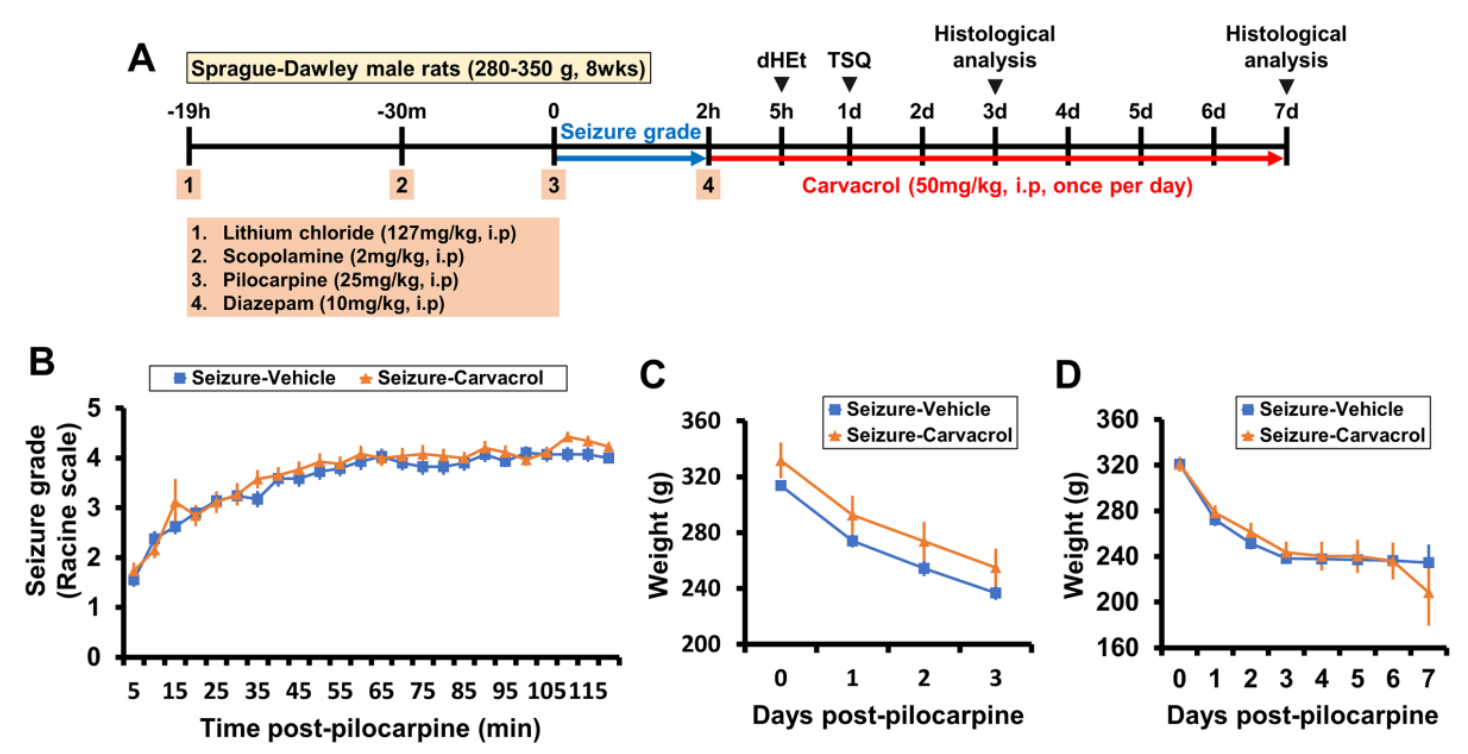

Figure 1. Carvacrol did not affect body weight following pilocarpine-induced status epilepticus (SE). (A) Timeline showing the experimental procedures. SE was induced by lithium-pilocarpine injection. Carvacrol was intraperitoneally administered once per day for all experimental periods. (B) Line graphs showing the seizure grade based on the Racine stage during SE induction by pilocarpine (mean \pm SEM; $n=26-29$ per group). (C,D) Graphs representing the weight change for 3 (C) or 7 days (D) after SE (mean \pm SEM; $n=8-10$ per group).

To investigate whether carvacrol could inhibit the TRPM7 channel after seizure, we used immunofluorescence staining to analyze the expression of TRPM7. Like previous studies, we found that TRPM7 exists primarily on cell bodies and is processed in hippocampal CA1 pyramidal neurons. In the sham-operated groups, there was no significant difference in TRPM7 expression between the vehicle- and carvacrol-treated groups. The vehicle-treated seizure group revealed a significant up-regulation of TRPM7 expression at 3 days following pilocarpine-induced SE. However, carvacrol treatment remarkably reduced TRPM7 expression after SE (sham-vehicle, $13.5 \pm 1.6$; sham-carvacrol, $13.2 \pm 1.5$, seizure-vehicle, $32.6 \pm 1.7$; seizure-carvacrol, $22.7 \pm 1.8$, a 30.4\% decrease; Figure $2 \mathrm{~A}, \mathrm{~B}$ ). 
Western blot also revealed a significant increase in the level of TRPM7 protein in the hippocampus $24 \mathrm{~h}$ after pilocarpine-induced SE as compared with the sham-operated groups. However, administration of carvacrol significantly decreased the protein level of TRPM7, compared to the vehicle-treated SE group (sham-vehicle, $1 \pm 0.07$; sham-carvacrol, $0.994 \pm 0.08$, seizure-vehicle, $1.412 \pm 0.15$; seizure-carvacrol, $0.965 \pm 0.07$, a 31.7\% decrease; Figure 2C,D). Next, to examine the effects of carvacrol on seizure-induced zinc accumulation and neuronal degeneration, we performed TSQ (6-methoxy-8-p-toluenesulfonamido-quinoline) and Fluoro Jade-B (FJB) staining on the brains harvested $24 \mathrm{~h}$ and 3 days after SE, respectively. TSQ, which can bind free or loosely bound zinc, is a commonly used zinc sensor [56-58], and FJB is useful for the detection of degenerating neurons [59]. SE induced by lithium-pilocarpine led to considerable accumulation of intracellular free zinc and neuronal death in hippocampal CA1. Compared to the vehicle-treated group, the number of $\mathrm{TSQ}^{+}$neurons was remarkably decreased in the carvacrol-treated group (seizure-vehicle, $56 \pm 5.6$; seizure-carvacrol, $20 \pm 6.6$, a $64.3 \%$ decrease; Figure 2E,F). In addition, FJB staining also showed that carvacrol treatment significantly reduced the number of degenerating neurons (seizure-vehicle, $197 \pm 26.5$; seizure-carvacrol, $117 \pm 20$, a 40.6\% decrease; Figure $2 \mathrm{G}, \mathrm{H}$ ). These findings indicate that carvacrol treatment reduces the accumulation of intracellular free zinc by inhibiting the overexpression of the TRPM7 channel, which occurs in the hippocampal CA1 following pilocarpine-induced SE, thereby causing a reduction in neuronal degeneration.
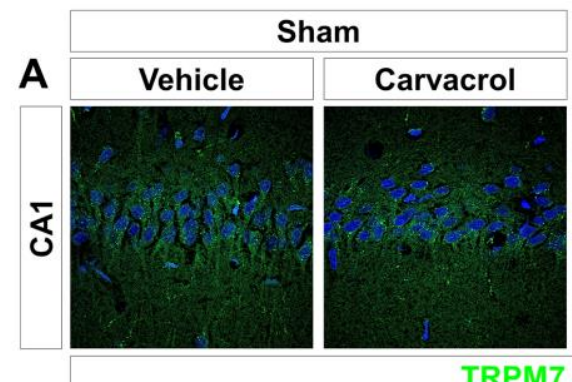

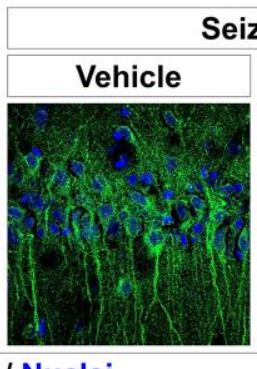

TRPM7 / Nuclei

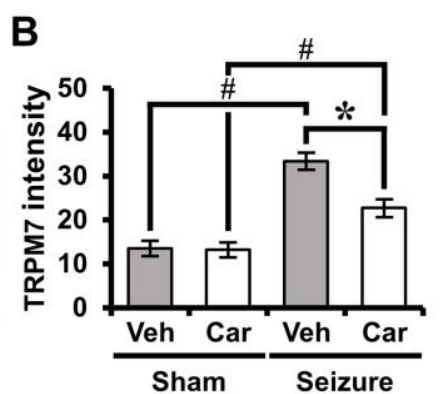

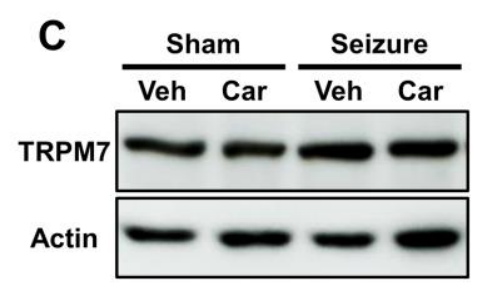

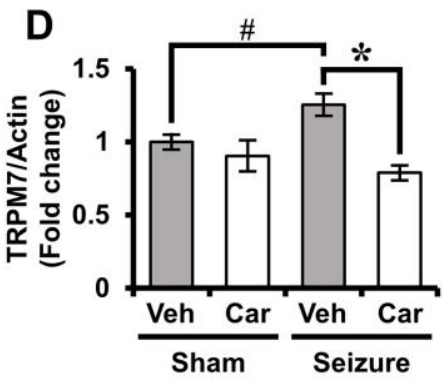

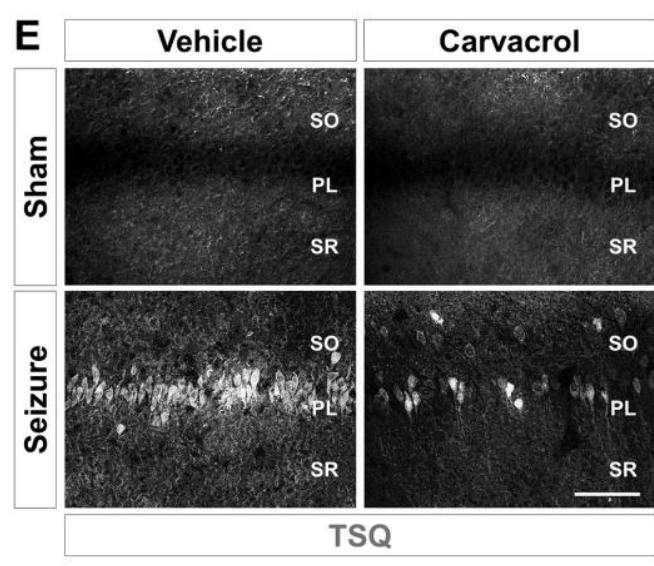

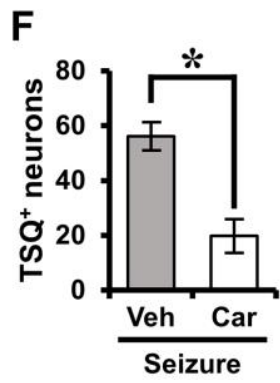

Figure 2. Cont. 

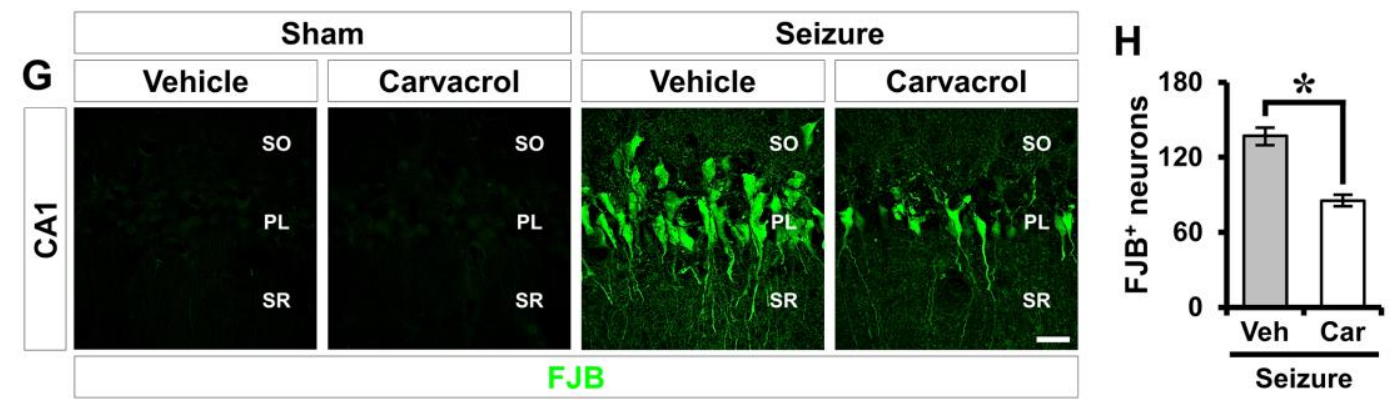

Figure 2. Carvacrol treatment reduces TRPM7 overexpression, zinc accumulation, and neuronal degeneration after seizure. (A) Representative images showing TRPM7 immunoreactivity (green) in the CA1 of the hippocampus. Nuclei are counterstained with DAPI (blue). Scale bar $=20 \mu \mathrm{m}$. (B) The bar graph representing the immunofluorescence intensity of TRPM7 as determined in the same hippocampal region (mean \pm SEM; $n=5$ from each sham group, $n=7$ from each seizure group). * $p<0.05$ vs. the vehicle-treated group; \# $p<0.05$ vs. the sham-operated group (Kruskal-Wallis test followed by a Bonferroni post-hoc test: chi square $=19.452, \mathrm{df}=3, p<0.001$ ). (C) Western blot analysis of TRPM7 in the hippocampus. (D) Quantification of TRPM7 protein levels from the hippocampus. (mean $\pm \mathrm{SEM} ; n=5$ from each sham group, $n=7-8$ from each seizure group). ${ }^{*} p<0.05$ vs. the vehicle-treated group; \# $p<0.05$ vs. the sham-operated group (Kruskal-Wallis test followed by a Bonferroni post-hoc test: chi square $=12.310, \mathrm{df}=3, p<0.006$ ). (E) Representative images showing sections of the hippocampus stained with TSQ to detect zinc accumulation. Scale bar $=100 \mu \mathrm{m}$. (F) Bar graph showing the number of $\mathrm{TSQ}^{+}$neurons in the hippocampal CA1 (mean $\pm \mathrm{SEM} ; n=6$ from each sham group, $n=5$ from each seizure group). ${ }^{*} p<0.05$ vs. the vehicle-treated group (Mann-Whitney $\mathrm{U}$ test: $z=2.611, p=0.008)$. (G) Fluorescent images representing the degenerating neurons stained with Fluoro-Jade B (FJB) in the hippocampal CA1. Scale bar $=20 \mu \mathrm{m}$. (H) Quantification of the number of $\mathrm{FJB}^{+}$neurons (mean $\pm \mathrm{SEM} ; n=5$ from each sham group, $n=8$ from each seizure group). ${ }^{*} p<0.05$ vs. the vehicle-treated group (Mann-Whitney $\mathrm{U}$ test: $z=1.785, p=0.074$ ).

\subsection{Carvacrol Reduces Seizure-Induced Superoxide Production and Oxidative Stress}

To determine whether carvacrol could affect reactive oxygen species (ROS) production and oxidative stress in the hippocampal CA1 following pilocarpine-induced SE, we analyzed Et fluorescence and immunofluorescence by dihydroethidium (dHEt) and 4-hydroxynonenal (4HNE) staining, respectively. $\mathrm{dHEt}$ and $4 \mathrm{HNE}$ are used as indicators of ROS or lipid peroxidation, respectively. SE induced by lithium-pilocarpine gave rise to increased Et fluorescence and 4HNE intensity in the hippocampal CA1. Carvacrol treatment remarkably reduced the intensity of the Et signal and 4HNE immunofluorescence after SE compared to the vehicle-treated SE group (Figure 3A,B: sham-vehicle, $4.3 \pm 1.7$; sham-carvacrol, $5 \pm 1.3$, seizure-vehicle, $45.5 \pm 7.9$; seizure-carvacrol, $19.5 \pm 6.1$, a $57.1 \%$ decrease; Figure 3C,D: sham-vehicle, $2.6 \pm 0.6$; sham-carvacrol, $1.8 \pm 0.2$, seizure-vehicle, $21.6 \pm 1.2$; seizure-carvacrol, $13.5 \pm 1.9$, a $37.5 \%$ decrease).
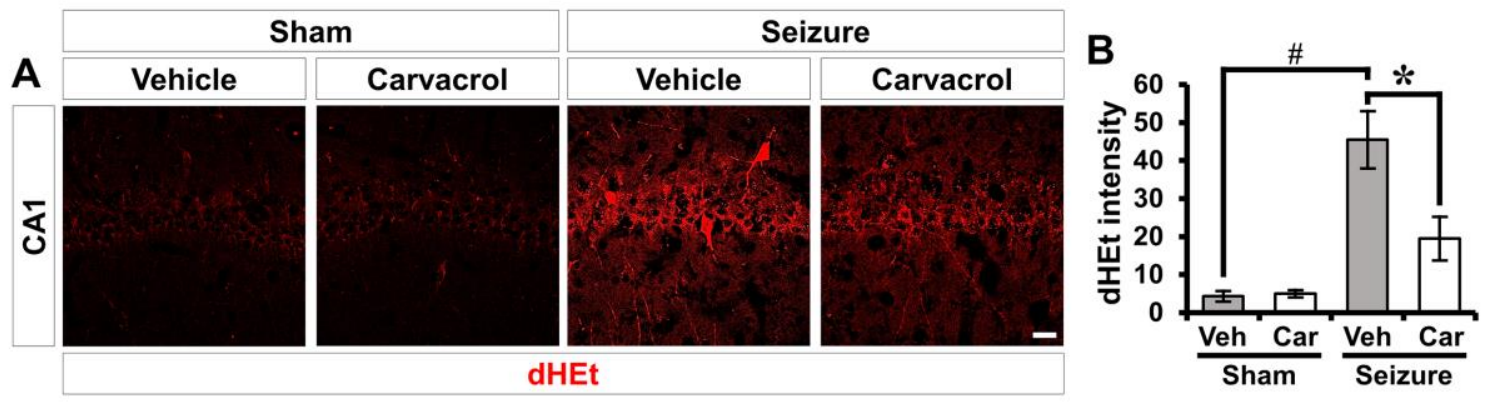

Figure 3. Cont. 

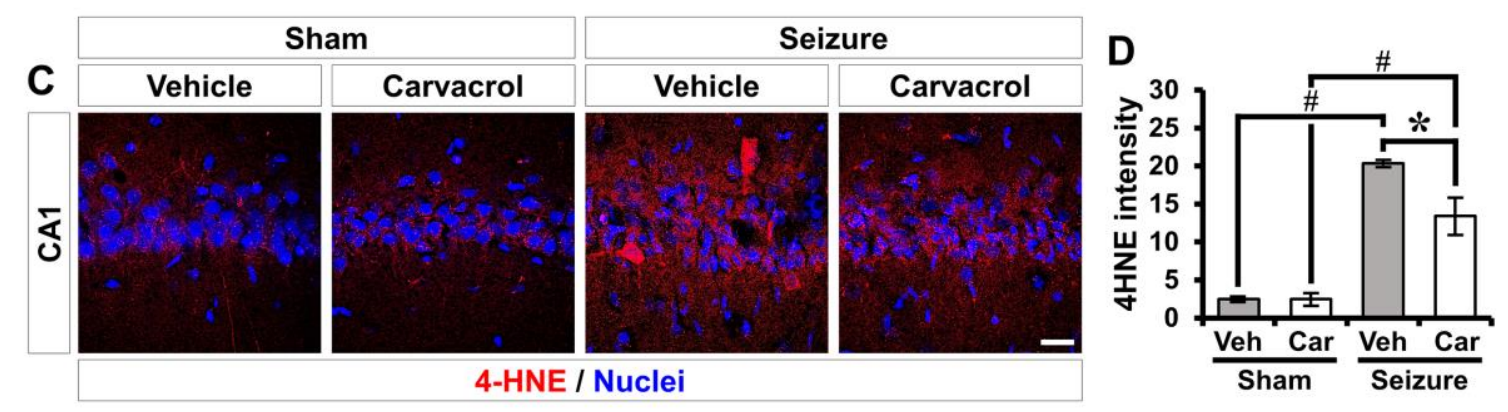

Figure 3. Carvacrol treatment reduces reactive oxygen species (ROS) production and oxidative stress after seizure. (A) Representative images showing the Et fluorescence in the hippocampal CA1. Scale bar $=20 \mu \mathrm{m}$. (B) The bar graph representing the intensity of Et fluorescence as determined in the same hippocampal region (mean $\pm \mathrm{SEM} ; n=6$ from each sham group, $n=5-6$ from each seizure group). ${ }^{*} p<0.05$ vs. the vehicle-treated group (Kruskal-Wallis test followed by a Bonferroni post-hoc test: chi square $=16.579, \mathrm{df}=3, p<0.001)$. (C) Immunofluorescent images representing the lipid peroxidation stained for anti-4HNE (red) in the hippocampal CA1. Nuclei are counterstained with DAPI (blue). Scale bar $=20 \mu \mathrm{m}$. (D) Quantification of the immunofluorescence intensity of $4 \mathrm{HNE}$ as determined in the same hippocampal region (mean \pm SEM; $n=5$ from each sham group, $n=7$ from each seizure group). ${ }^{*} p<0.05$ vs. the vehicle-treated group; $\# p<0.05$ vs. the sham-operated group (Kruskal-Wallis test followed by a Bonferroni post-hoc test: chi square $=19.853, \mathrm{df}=3, p<0.001$ ).

\subsection{Carvacrol Reduces Glial Activation after Pilocarpine-Induced Seizure}

We next assessed the effects of carvacrol treatment on microglia/macrophage activation following pilocarpine-induced SE. The activation of microglia and macrophages was monitored by double immunofluorescence staining using antibodies against the ionized calcium-binding adapter molecule 1 (Iba-1) and the cluster of differentiation 68 (CD68). Iba-1 is constitutively expressed in microglia/macrophages, and CD68 is a lysosome-associated membrane protein. Colocalization with Iba-1 and CD68 is well known as an indicator of microglia/macrophage M1 polarization [60]. The sham-operated groups showed ramified microglia/macrophage morphology in a resting state. The Iba-1 immunoreactivity in the vehicle-treated sham group was similar to that in the carvacrol-treated sham group. The vehicle-treated SE group had prominent microglia/macrophage activation in the hippocampal CA1. In addition, many Iba- $1^{+}$microglia/macrophages were significantly co-localized with CD68 at 3 days following pilocarpine-induced SE. In contrast, carvacrol treatment decreased the intensity of Iba-1 and CD68 immunofluorescence after SE (sham-vehicle, $6.9 \pm 1.5$; sham-carvacrol, $5.1 \pm 0.6$, seizure-vehicle, $42.8 \pm 4.7$; seizure-carvacrol, $28.1 \pm 1.2$, a $34.3 \%$ decrease, sham-vehicle, $3.3 \pm 0.6$; sham-carvacrol, $3.1 \pm 0.4$, seizure-vehicle, $31.5 \pm 3.3$; seizure-carvacrol, $22.5 \pm 2.3$, a $28.6 \%$ decrease; Figure $4 \mathrm{~A}-\mathrm{C}$ ). We also investigated whether carvacrol could decrease astroglial activation following pilocarpine-induced SE. The activation of astrocyte was monitored by immunofluorescence staining using an antibody against the astrocyte-specific marker glial fibrillary acidic protein (GFAP). Three days after SE, the GFAP ${ }^{+}$astrocyte in the vehicle-treated SE group clearly showed hypertrophied processes, which indicates the development of astrogliosis and the activation of astrocytes. However, carvacrol treatment remarkably decreased astrogilal activation in the hippocampus after SE (sham-vehicle, $7.4 \pm 1.4$; sham-carvacrol, $3.7 \pm 0.3$, seizure-vehicle, $24.1 \pm 1.5$; seizure-carvacrol, $11.9 \pm 2.5$, a 50.6\% decrease; Figure 4D,E). 
A

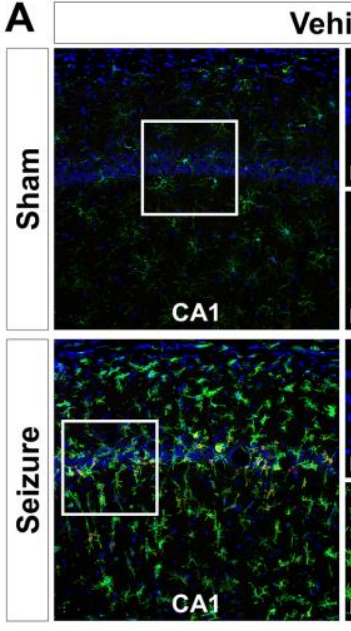

B
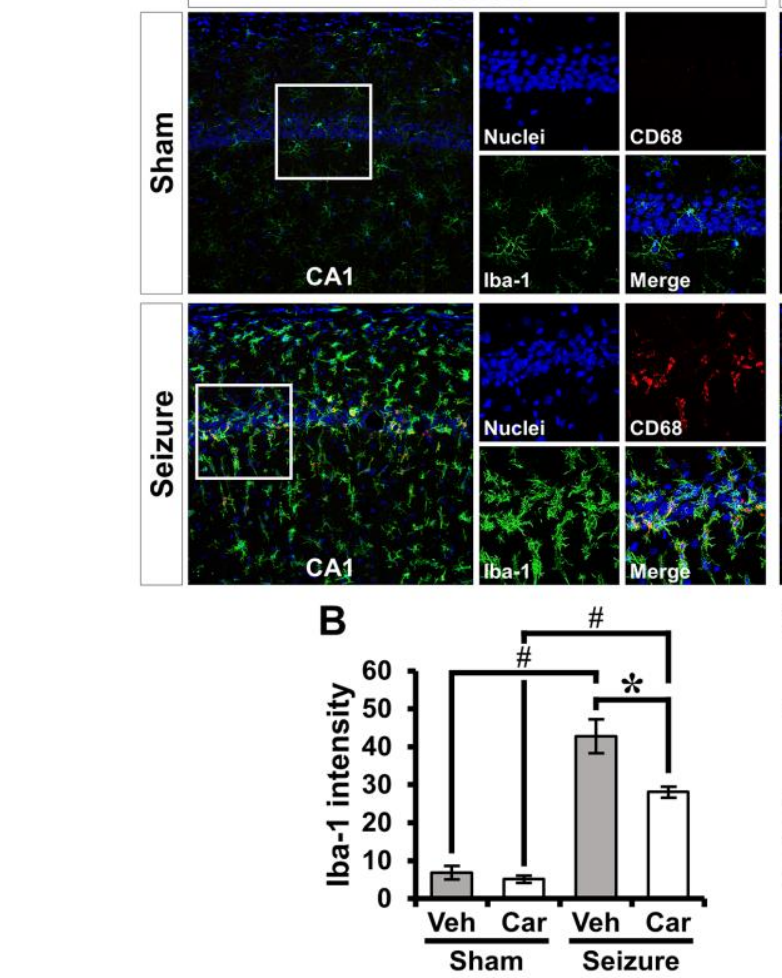

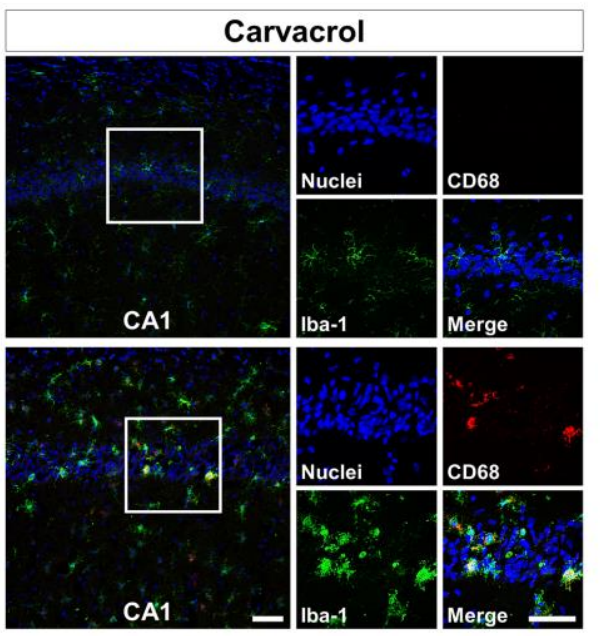

C

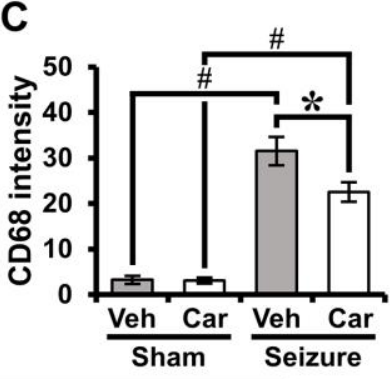

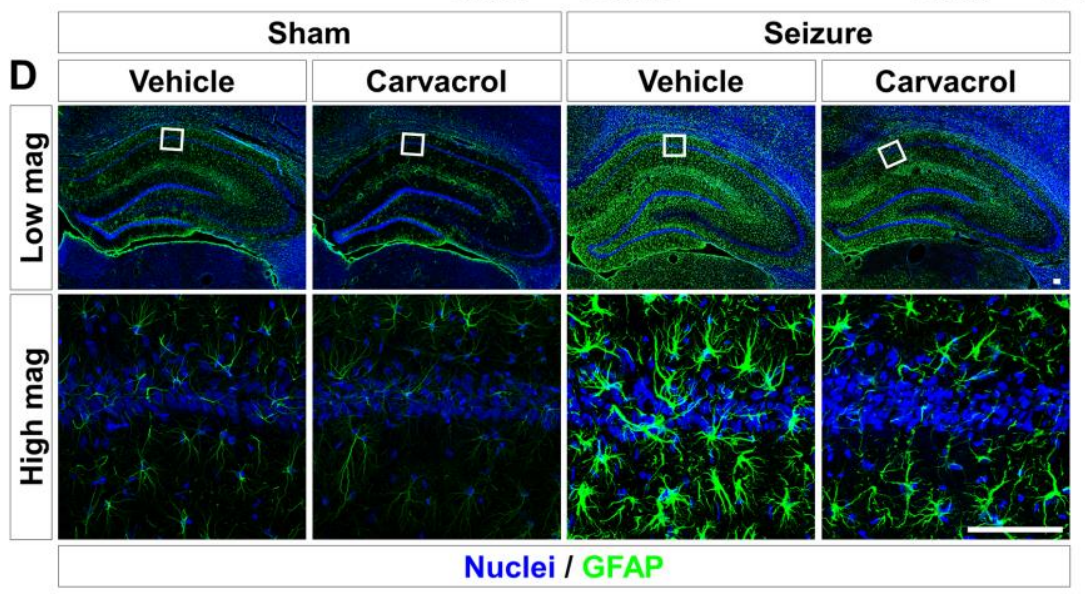

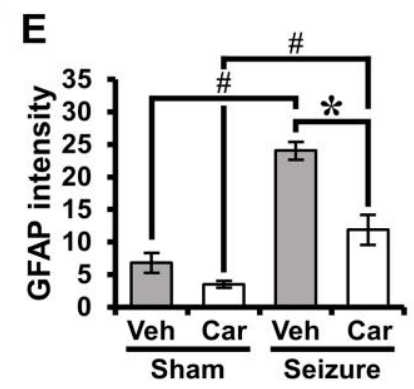

Figure 4. Carvacrol treatment reduces seizure-induced glial activation. (A) Double-label confocal micrographs of Iba-1 (green) and CD68 (red) in the hippocampal CA1 from the vehicle- and carvacrol-treated groups after sham or SE. Nuclei stained with DAPI (blue). Scale bar, $50 \mu \mathrm{m}$. (B,C) Bar graphs showing the intensity of Iba-1 (B) and CD68 (C) as determined in the same hippocampal region (mean $\pm \mathrm{SEM} ; n=5$ from each sham group, $n=6-8$ from each seizure group). ${ }^{*} p<0.05$ vs. the vehicle-treated group; \# $p<0.05$ vs. the sham-operated group (Kruskal-Wallis test followed by a Bonferroni post-hoc test; Figure 4B: chi square $=19.244, \mathrm{df}=3, p<0.001$; Figure $4 \mathrm{C}$ : chi square $=18.708$, $\mathrm{df}=3, p<0.001$ ). (D) Immunofluorescent images representing the astrocyte activation stained for anti-GFAP (green) in the hippocampus. Nuclei are counterstained with DAPI (blue). Scale bar $=100 \mu \mathrm{m}$. (E) Quantification of the immunofluorescence intensity of GFAP as determined in the same hippocampal region (mean \pm SEM; $n=4-5$ from each sham group, $n=7$ from each seizure group). ${ }^{*} p<0.05$ vs. the vehicle-treated group; \# $p<0.05$ vs. the sham-operated group (Kruskal-Wallis test followed by a Bonferroni post-hoc test: chi square $=16.32, \mathrm{df}=3, p=0.001$ ). 


\subsection{Carvacrol Reduces Seizure-Induced BBB Breakdown and Vessel Disorganization}

To analyze the putative blood-brain barrier (BBB) damage, we sought to confirm the leakage of serum IgGs using immunohistochemistry. In the sham-operated groups, there was little or no leakage of the IgGs, whereas, in the pilocarpine-induced SE groups, we observed a significant increase in IgG immunoreactivity throughout the hippocampus. Compared to the vehicle-treated SE group, IgG immunoreactivity was significantly reduced in in the hippocampus of the carvacrol-treated SE group (sham-vehicle, $1 \pm 0.2$; sham-carvacrol, $1.1 \pm 0.2$, seizure-vehicle, $2.7 \pm 0.1$; seizure-carvacrol, $2.0 \pm 0.1$, a $25.9 \%$ decrease; Figure 5A,B). We also checked the extravasation of endogenous serum IgGs from vessels following pilocarpine-induced SE. Sections were stained for endogenous IgG and the endothelial protein found in areas with BBB (SMI-71), which can be used as an indicator of BBB damage, to highlight vascular permeability. The vehicle-treated SE group showed pronounced and diffuse IgG immunoreactivity in the vessels of the hippocampus, reflecting endogenous serum protein extravasation, which obscured the boundaries between the vessel segments. However, carvacrol treatment significantly decreased IgG immunoreactivity in the vessels, which suggests that the carvacrol preserved the integrity of the BBB (Figure 5C). In addition, we examined the distribution of SMI-71 ${ }^{+}$blood vessels in the hippocampus at 3 days post SE. The SMI-71+ ${ }^{+}$essels were widely distributed throughout the hippocampus. The vehicle-treated SE group showed a considerable disappearance of SMI-71 blood vessels, particularly in the molecular layer of the dentate gyrus (DG) and the hippocampal CA1 subfield. By contrast, carvacrol treatment significantly inhibited the disappearance of SMI-71 ${ }^{+}$vessels in the hippocampus (sham-vehicle, $26.8 \pm 3$; sham-carvacrol, $23.5 \pm 0.7$, seizure-vehicle, $10.8 \pm 2.3$; seizure-carvacrol, $19.6 \pm 0.7$, a $81 \%$ increase; Figure 5D,E). These results indicate that carvacrol treatment preserved BBB integrity and inhibited vessel disorganization following pilocarpine-induced SE.
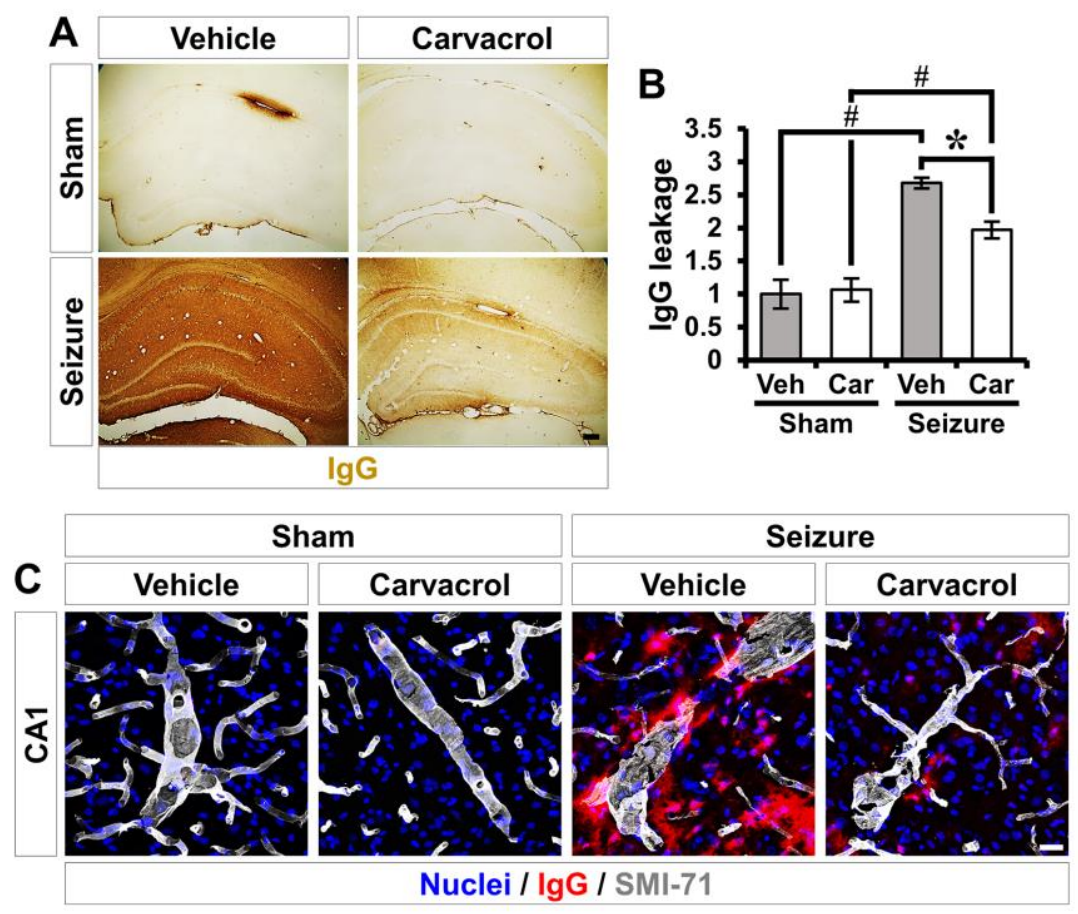

Figure 5. Cont. 

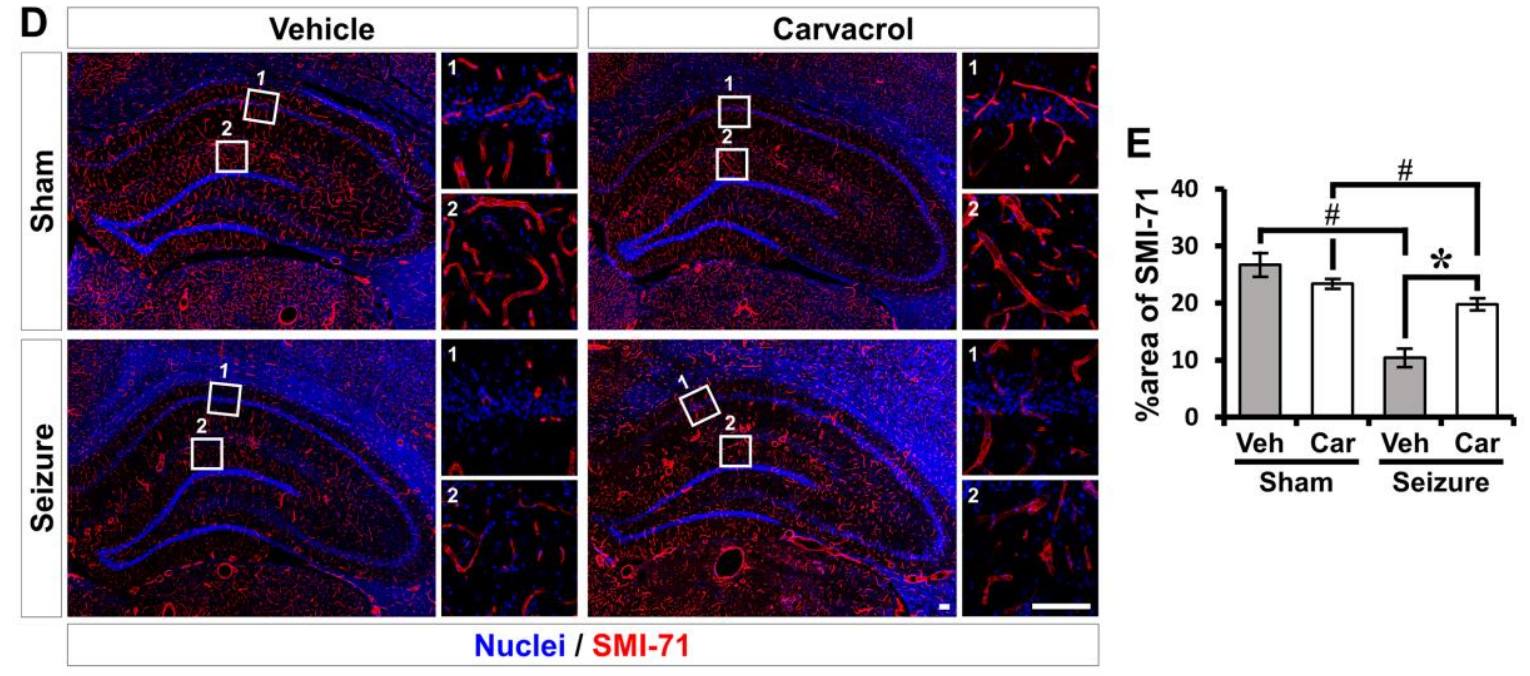

Figure 5. Carvacrol treatment reduces the blood-brain barrier (BBB) disruption and vessel disappearance following pilocarpine-induced SE. (A) Photomicrographs showing sections of the hippocampus stained for anti-mouse immunoglobulin G (IgG) to detect endogenous IgG. Scale bar, $100 \mu \mathrm{m}$. (B) Graph representing IgG leakage from the hippocampus in mice treated with the vehicle or carvacrol at 3 days following pilocarpine-induced SE (mean \pm SEM; $n=5$ from each sham group, $n=7$ from each seizure group). ${ }^{*} p<0.05$ vs. the vehicle-treated group; $\# p<0.05$ vs. the sham-operated group (Kruskal-Wallis test followed by Bonferroni post-hoc test; chi square $=18.493, \mathrm{df}=3, p<0.001$ ). (C) Double Immunofluorescent images representing the BBB marker SMI-71+ endothelial protein (gray) and endogenous IgG leakage (red) in the hippocampus. Nuclei are counterstained with DAPI (blue). Scale bar $=20 \mu \mathrm{m}$. (D) Representative images showing the SMI- $71^{+}$endothelial protein (red) in the hippocampus. Nuclei are counterstained with DAPI (blue). Scale bar, $100 \mu \mathrm{m}$. (E) Quantification of the percent area of the SMI-71+ endothelial protein in the hippocampus (mean \pm SEM; $n=3-4$ from each sham group, $n=5-7$ from each seizure group). ${ }^{*} p<0.05$ vs. the vehicle-treated group; $\# p<0.05$ vs. the sham-operated group (Kruskal-Wallis test followed by a Bonferroni post-hoc test: chi square $=19.080$, $\mathrm{df}=3, p<0.001)$.

\subsection{Carvacrol Reduces Seizure-Induced Apoptotic Neuronal Death}

To assess whether carvacrol has neuroprotective effects, we analyzed the delayed neuronal loss at 7 days following pilocarpine-induced SE. The sham-operated groups showed intense staining of the neuronal nuclei $(\mathrm{NeuN})^{+}$neurons in the hippocampal CA1. There was no significant difference in the number of $\mathrm{NeuN}^{+}$neurons between the vehicle- and carvacrol-treated groups. Pilocarpine-induced $\mathrm{SE}$ led to a significant reduction in the number of $\mathrm{NeuN}^{+}$neurons compared to the sham-operated groups. The number of $\mathrm{NeuN}^{+}$neurons was remarkably higher in the carvacrol-treated group than in the vehicle-treated group (sham-vehicle, $429 \pm 13.9$; sham-carvacrol, $416 \pm 11.9$, seizure-vehicle, $171 \pm 10.5$; seizure-carvacrol, $209 \pm 13.3$, a 22\% increase; Figure 6A,B). We next asked how carvacrol protects hippocampal CA1 pyramidal neurons by determining the differences in the apoptotic neuronal death in the vehicle- and carvacrol-treated SE groups. To examine this, we conducted double immunofluorescence staining using antibodies against NeuN and cleaved caspase-3, which is an indicator of cell death during apoptosis. The number of caspase- $3^{+}$and $\mathrm{NeuN}^{+}$caspase- $3^{+}$cells was significantly reduced in the carvacrol-treated SE group compared to the vehicle-treated SE group. We also found that carvacrol treatment revealed a significant decrease in the percentage of NeuN+Caspase- $3+$ cells among $\mathrm{NeuN}^{+}$cells after SE compared to the vehicle-treated SE group (seizure-vehicle, $383 \pm 22.5$; seizure-carvacrol, $262 \pm 34.7$, a 31.6\% decrease, seizure-vehicle, $71 \pm 11.8$; seizure-carvacrol, $31 \pm 8.4$, a $56.3 \%$ decrease, seizure-vehicle, $55 \pm 5.4$; seizure-carvacrol, $19 \pm 6.3$, a $65.5 \%$ decrease; Figure $6 \mathrm{C}-\mathrm{F}$ ). These results suggest that carvacrol reduces apoptotic neuronal death following pilocarpine-induced SE. 

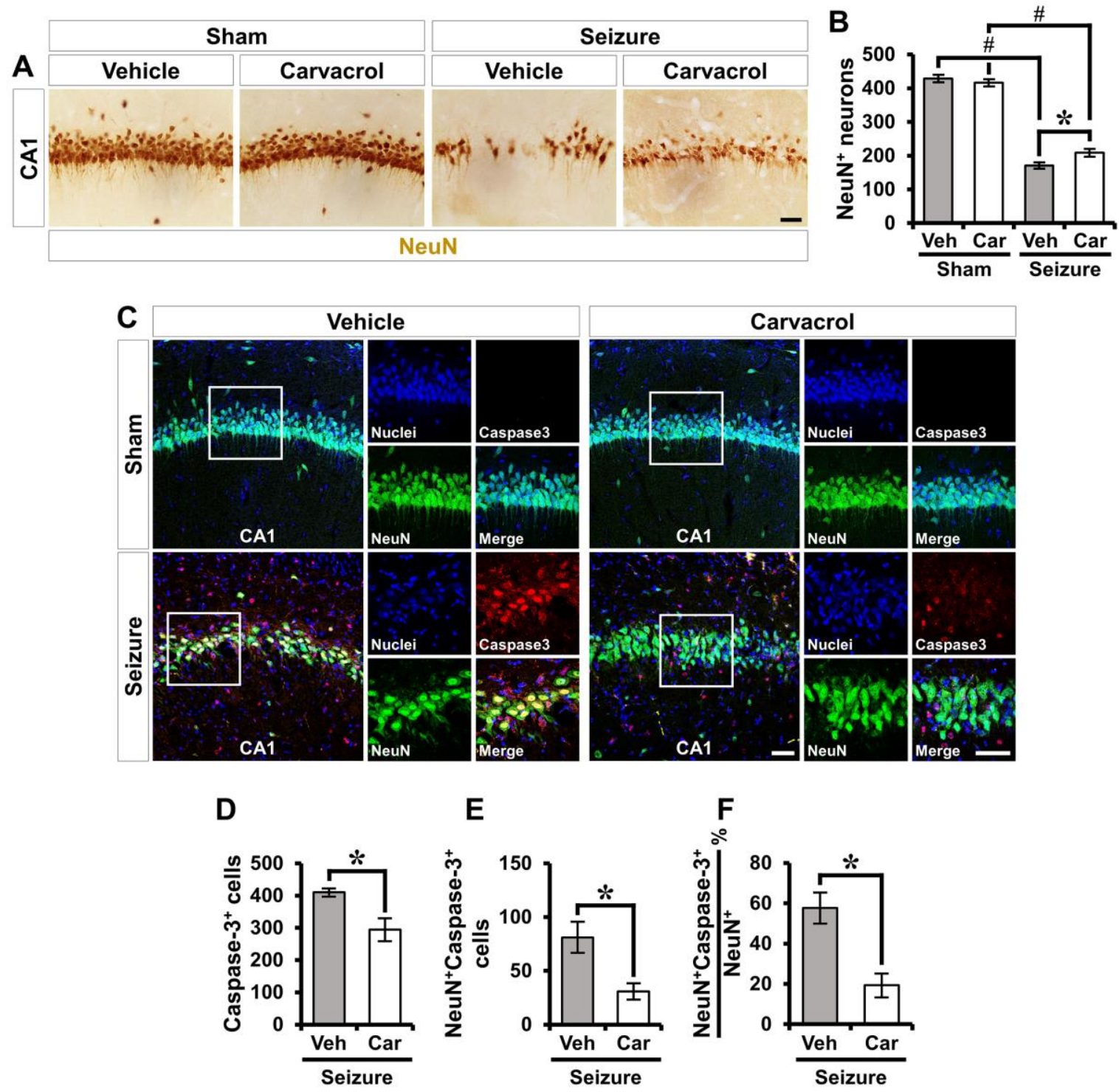

Figure 6. Carvacrol treatment reduces apoptotic neuronal death following pilocarpine-induced SE. (A) Representative images showing the expression of $\mathrm{NeuN}^{+}$live neurons in the hippocampal CA1 from the vehicle- and carvacrol-treated groups 1 week after sham or SE. Scale bar, $100 \mu \mathrm{m}$. (B) Quantification showing the number of $\mathrm{NeuN}^{+}$neurons as determined in the same hippocampal region (mean $\pm \mathrm{SEM}$; $n=6$ from each sham group, $n=10$ from each seizure group). ${ }^{*} p<0.05$ vs. the vehicle-treated group; \# $p<0.05$ vs. the sham-operated group (Kruskal-Wallis test followed by a Bonferroni post-hoc test; chi square $=23.958, \mathrm{df}=3, p<0.001$ ). (C) Double immunofluorescent images representing the neuronal marker $\mathrm{NeuN}^{+}$cells (green) co-labeled with the cleaved caspase-3 (red) in the hippocampal CA1 from the vehicle- and carvacrol-treated groups after SE. The nuclei are counterstained with DAPI (blue). Scale bar, $50 \mu \mathrm{m}$. (D-F) Quantification of the number of caspase- $3^{+}$(D) and $\mathrm{NeuN}^{+}$Caspase- $3^{+}$ cells (E) and the percent of $\mathrm{NeuN}^{+}$Capase- $3^{+}$cells over total $\mathrm{NeuN}^{+}$cells $(\mathbf{F})$ as determined in the same hippocampal region (mean $\pm \mathrm{SEM} ; n=4-6$ per group). ${ }^{*} p<0.05$ vs. the vehicle-treated group (Mann-Whitney U test: Figure 6D: $z=2.082, p=0.041$; Figure 6E: $z=2.242, p=0.026$; Figure 6F: $z=2.882, p=0.002$ ).

\subsection{2-APB Reduces Seizure-Induced TRPM7 Overexpression, Zinc Accumulation, and Neuronal Death}

To further confirm our initial results, we tested whether 2-APB, another non-specific inhibitor of TRPM7, promoted neuroprotective effects after seizure with a pilocarpine-induced SE model. To investigate whether 2-APB could alter the expression of the TRPM7 channel after seizure, we used 
immunofluorescence staining to analyze surface expression of TRPM7. In the sham-operated groups, there was no significant difference in TRPM7 expression between the vehicle- and carvacrol-treated groups. The vehicle-treated seizure group revealed a significant up-regulation of TRPM7 expression at 3 days following pilocarpine-induced SE. However, carvacrol treatment remarkably reduced TRPM7 expression after SE (sham-vehicle, $2.1 \pm 0.4$; sham-carvacrol, $3 \pm 0.3$, seizure-vehicle, $22.8 \pm 1.0$; seizure-carvacrol, $16.7 \pm 1.8$, a 26.8\% decrease; Figure 7A,B). To determine whether 2-APB affects zinc accumulation and neuronal death after seizure, we performed TSQ (6-methoxy-8-p-toluenesulfonamido-quinoline) and Fluoro-Jade B (FJB) staining. SE induced by lithium-pilocarpine led to considerable accumulation of intracellular free zinc and neuronal death in hippocampal CA1. Compared to the vehicle-treated group, the number of $\mathrm{TSQ}^{+}$neurons was remarkably decreased in the 2-APB-treated group (seizure-vehicle, $37 \pm 8.2$; seizure-carvacrol, $9 \pm 2.3$, a 75.7\% decrease; Figure 7C,D). In addition, FJB staining also showed that 2-APB treatment significantly reduced the number of degenerating neurons (seizure-vehicle, $204 \pm 17.5$; seizure-carvacrol, $115 \pm 11.2$, a $43.6 \%$ decrease; Figure $7 \mathrm{E}, \mathrm{F})$. These findings indicate that 2-APB treatment also reduces the accumulation of intracellular free zinc by inhibiting the overexpression of the TRPM7 channel, which occurs in the hippocampal CA1 following pilocarpine-induced SE, thereby causing a reduction in neuronal degeneration.
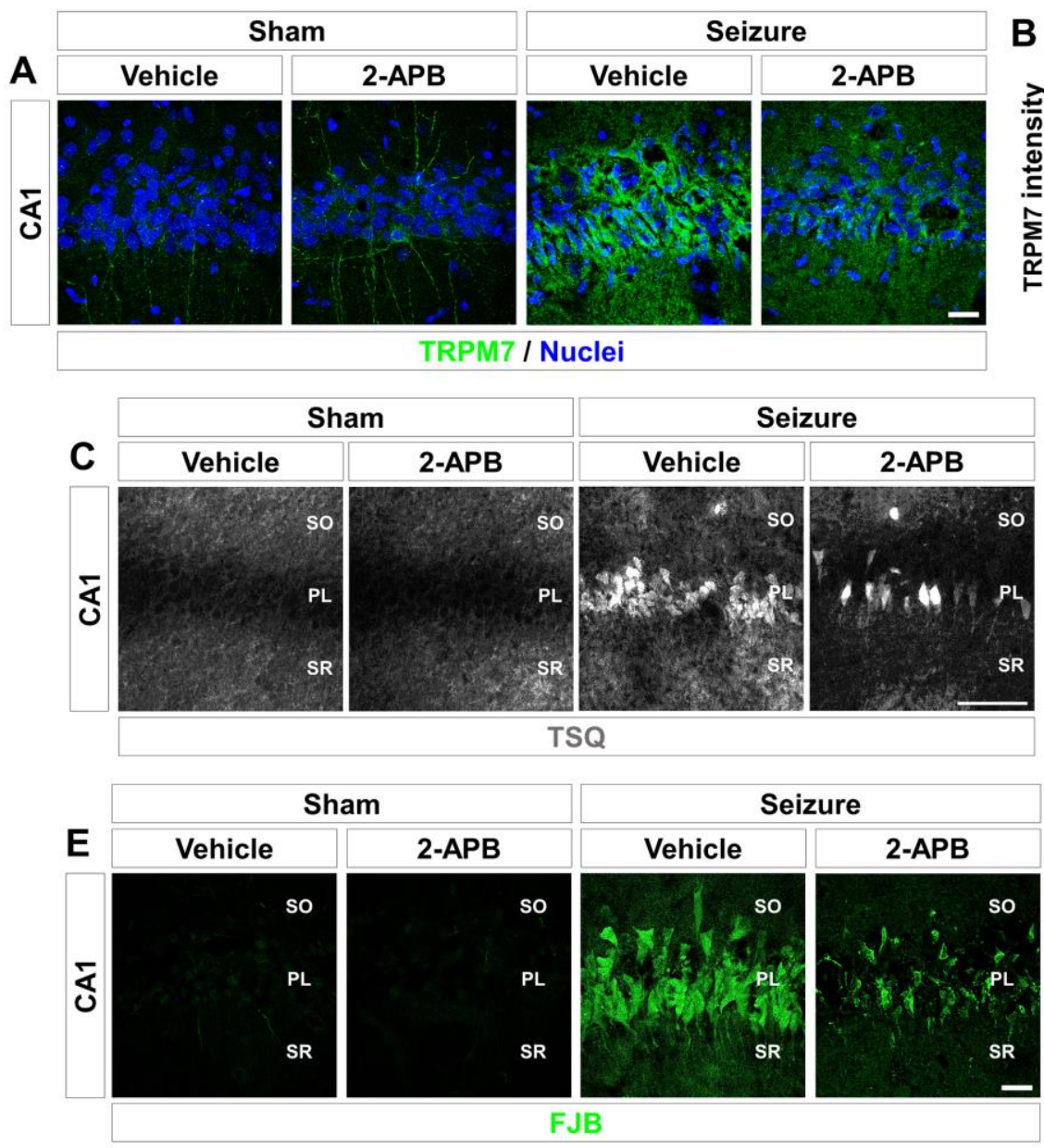
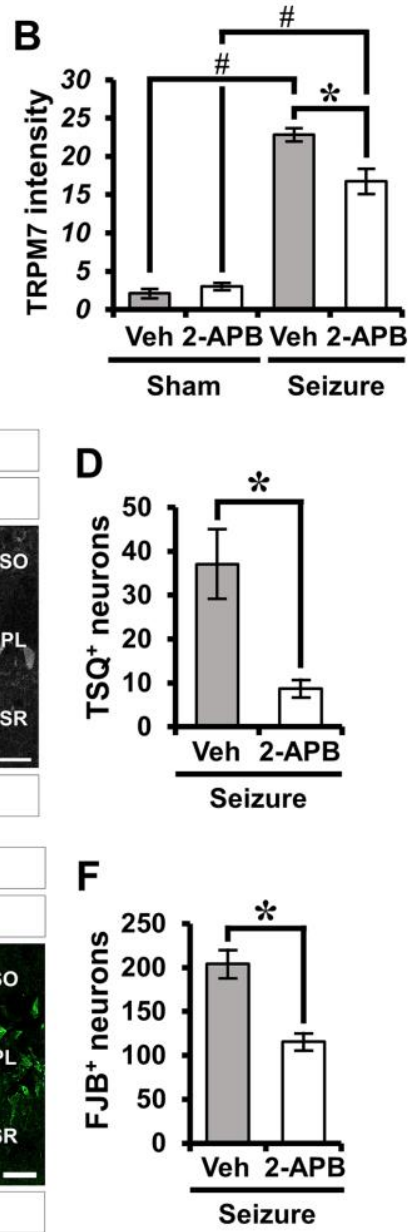

Figure 7. 2-APB treatment reduces TRPM7 overexpression, zinc accumulation, and neuronal degeneration after seizure. (A) Representative images showing TRPM7 immunoreactivity (green) in the CA1 of the hippocampus. Nuclei are counterstained with DAPI (blue). Scale bar $=20 \mu \mathrm{m}$. (B) The bar graph representing the immunofluorescence intensity of TRPM7 as determined in the same hippocampal 
region (mean \pm SEM; $n=5$ from each sham group, $n=5-6$ from each seizure group). ${ }^{*} p<0.05$ vs. the vehicle-treated group; \# $p<0.05$ vs. the sham-operated group (Kruskal-Wallis test followed by a Bonferroni post-hoc test: chi square $=17.451, \mathrm{df}=3, p<0.001)$. (C) Representative images showing sections of the hippocampus stained with TSQ to detect zinc accumulation. Scale bar $=100 \mu \mathrm{m}$. (D) Bar graph showing the number of $\mathrm{TSQ}^{+}$neurons in the hippocampal CA1 (mean $\pm \mathrm{SEM} ; n=6$ from each sham group, $n=5-6$ from each seizure group). ${ }^{*} p<0.05$ vs. the vehicle-treated group (Mann-Whitney $\mathrm{U}$ test: $z=2.739, p=0.004$ ). (E) Fluorescent images representing the degenerating neurons stained with Fluoro-Jade B (FJB) in the hippocampal CA1. Scale bar $=20 \mu \mathrm{m}$. (F) Quantification of the number of $\mathrm{FJB}^{+}$neurons (mean $\pm \mathrm{SEM} ; n=5$ from each sham group, $n=5-6$ from each seizure group). ${ }^{*} p<0.05$ vs. the vehicle-treated group (Mann-Whitney U test: $z=2.373, p=0.017$ ).

\section{Discussion}

Using lithium-pilocarpine-induced SE as a model for TLE, we investigated the therapeutic potential of carvacrol to protect against hippocampal neuron death induced by the zinc-mediated pathogenic mechanisms following SE. Here, we found that carvacrol reduced seizure-induced TRPM7 overexpression, intracellular zinc accumulation, and ROS production. Moreover, we observed the suppression of oxidative stress, glial activation, and BBB breakdown. In addition, carvacrol remarkably decreased apoptotic neuron death following seizure. Thus, the neuroprotective effects of carvacrol in pilocarpine-induced SE may occur through the inhibition of TRPM7 and the subsequent reduction in intracellular free zinc accumulation.

Zinc plays an important role in maintaining the immune system, metabolic homeostasis, and antioxidant activity [61]. However, in pathological conditions such as TBI, ischemia, and seizure (which indicate a devastating state of the CNS), synaptically released zinc excessively enters into the intracellular space [62]. The accumulation of intracellular free zinc can induce neuronal death $[19,25,28,30]$.

TRPM7 is a selective cation permeable channel with serine/threonine kinase activity [63]. Divalent cations such as $\mathrm{Ca}^{2+}, \mathrm{Zn}^{2+}$, and $\mathrm{Mg}^{2+}$ that enter the cell through the TPRM7 channel play an important role in neuronal apoptosis [64]. However, among the cations that enter into the cell through the TRPM7 channel, zinc is known to have the highest affinity [65]. In addition, activation of the TRPM7 channel increases oxidative stress. Increased oxidative stress stimulates the TRPM7 channels more strongly, leading to divalent cation permeation through these channels $[35,66]$. A molecular study also showed that the TRPM7 channels affect zinc-mediated neurotoxicity [26,35]. Recently, Doboszewska et al. has suggested that the neuroprotective effects of carvacrol may be related to reduction of intracellular free zinc levels via inhibition of TRPM7 [26]. In the present study, we also hypothesize that inhibiting the TRPM7 channel is expected to inhibit zinc from entering the cell through this channel. Thus, the purpose of this study was to identify the effect of carvacrol on zinc-mediated neuron death after pilocarpine-induced SE. We first assessed TRPM7 expression to determine if carvacrol administration inhibited TRPM7 expression. As a result, we confirmed that overexpression of the TRPM7 channel increased after a seizure and decreased via the administration of carvacrol. Carvacrol may not directly down regulate TRPM7 protein expression but suppress a second round of up-regulation after initial expression. Seizures cause a significant increase in the level of free zinc in the hippocampus of the brain [62]. Among the cations that enter into the TRPM7 channel, zinc is known to have the highest affinity [33]. By inhibiting TRPM7 channels, the level of free zinc entering the cell can be reduced. In this way, it is possible to reduce the excessive accumulation of free zinc into the cell, which will reduce neuronal death. We found that zinc translocation and accumulation was reduced by carvacrol treatment. Because carvacrol is a non-specific inhibitor of the TRPM7 channel, we confirmed the effect of another non-specific inhibitor, 2-APB. 2-APB is known as an inhibitor of the TRP channel and an inhibitor of the IP3 receptor [52,67]. It has been reported that zinc toxicity in mouse cortical neurons was reduced by administration of 2-APB [35]. Therefore, we used 2-APB, another non-specific TRPM7 channel inhibitor, to confirm the protective effect of inhibiting TRPM7 activity on neuronal 
death after seizure. Administration of 2-APB after seizure reduced TRPM7 channel overexpression, zinc accumulation and neuronal death (Figure 7).

Oxidative injury, glial activation, and BBB disruption are involved in the process leading to neuronal death after seizures [68-70]. The excessive production of ROS gives rise to mitochondrial dysfunction and consequently resulted in neuronal death [71,72]. Antioxidant activity decreased after seizure, which also decreased antioxidant activity, thereby making the brain more susceptible to oxidative stress, as indicated by increased lipid peroxidation [73]. Numerous studies have demonstrated that zinc release is an upstream event of ROS production. The excessive activity of the TRPM7 channel and excessive accumulation of zinc also induced reactive oxygen species (ROS) [74,75]. The activated ROS acts to activate the TRPM7 channel and vice versa [74]. Since this vicious cycle greatly contributes to increasing neuronal death, we sought to reduce this phenomenon by inhibiting the TRPM7 channel via the administration of carvacrol. Here, we found that seizure-induced ROS production and oxidative injury were decreased by carvacrol administration. Our findings suggest that blocking TRPM7 by carvacrol reduces the accumulation of intracellular free zinc and thereby inhibits neuron death following pilocarpine-induced SE.

Microglia/macrophages are highly flexible glial cell of the CNS, which are recognized to play an important role not only in a healthy CNS but also in various pathological conditions [76]. In general, microglia/macrophages are present in a resting state under physiological conditions and are rapidly activated under pathological conditions, including increased chemokine and cytokine synthesis [77-79]. Activated microglia/macrophages gather around degenerating neurons in CNS disease. The first immune defense line, the microglia, responds rapidly to antigens, releasing tumor necrosis factor (TNF) $-\alpha$, interleukin (IL)-6, and nitric oxide (NO) [80,81]. Thus, microglia/macrophage activation is one of the most common early features of neurological diseases. Microglia/macrophage polarization (divided into M1 and M2) is the mediator of pro-inflammatory and anti-inflammatory responses, respectively [82]. The pro-inflammatory response promotes inflammatory cytokines, which are a type of signaling molecule released from immune cells, such as helper $\mathrm{T}$ cells and macrophages, as well as other cell types that promote inflammation [83]. The present study found that the activity of microglia/macrophages that leads to neuronal death following SE involves the activity of M1 microglia/macrophages and carvacrol treatment reduces the activation of M1 microglia/macrophages after SE.

$\mathrm{BBB}$, a unique anatomical and physiological interface between the CNS and peripheral circulation, is essential to provide a suitable environment for neurological function regardless of fluctuations in blood composition [84-86]. After epilepsy, pinocytotic activity and tight junction deformation increased and contributed to BBB dysfunction $[87,88]$. There are various mechanisms through which BBB dysfunction occurs after a seizure: free radical production, cytokine release, glutamate release, and inflammatory cytokines [89,90]. It is well known that BBB permeability increases after a seizure $[91,92]$. BBB breakdown can be caused by the activation of reactive oxygen species (ROS), matrix metalloproteinases (MMPs), inflammatory cytokines, immune cell extravasation, and leukocyte adhesion [89]. Impaired BBB integrity is mainly indicated by increased barrier permeability. Increased BBB permeability allows several cytokines to enter, such as IL-18 and TNF- $\alpha$, which are involved in the immune response [93]. In the present study, we showed that carvacrol treatment that has BBB permeability $[94,95]$ reduces seizure-induced BBB disruption by inhibiting the ROS, which increases permeability by destroying the BBB's integrity.

Oregano has a high content of carvacrol and thymol, which are phenolic antioxidants. Carvacrol is the most effective antifungal and antibacterial agent [96] while thymol is isomeric with carvacrol that strengthen the immune system [97]. However, there are several adverse side effects that can be expected besides weight loss, such as, toxicity due to inhibition of absorption of iron. Others include allergic reactions and die-off effects. If there is massive die-off, one might experience nausea and headache; digestive problems; blood thinning, tingling sensation in the mouth; high blood pressure in 
some people. In the present animal study, we did not find any of the above behavioral side effects in the carvacrol-treated rats during the entire experiment.

A previous study demonstrated that the administration of pilocarpine acts on the pre-synaptic and post-synaptic muscarinic receptors, thereby increasing the glutamate levels [98]. This leads to glutamate release from the presynaptic terminals. The released glutamate binds to the postsynaptic $\mathrm{N}$-methyl-D-aspartate receptor (NMDAR) and facilitates calcium influx into the cells [99]. Calcium enters into the cell through NMDAR and then stimulates the TRPM7 channel [100]. Zinc enters through the activated TRPM7 channel and accumulates [26]. Excessive zinc stimulates the ROS production produced by NADPH-oxidase and mitochondria, thereby triggering further intracellular zinc mobilization [101,102]. Thus, excessive zinc and ROS are capable of triggering signaling cascades that ultimately give rise to neuronal death by apoptosis $[103,104]$. We speculate that carvacrol and 2-APB may prevent initial translocation of zinc into postsynaptic neurons after seizure and then carvacrol reduced over expression of TRPM7 after seizure. The present study found that the inhibition of TRPM7 by carvacrol and 2-APB reduces zinc-mediated apoptotic neuron death following pilocarpine-induced SE (Figure 8).

\section{A. Hypothetical connection between zinc and neuronal death in epileptic seizure}

\section{B. Protective effects of carvacrol}
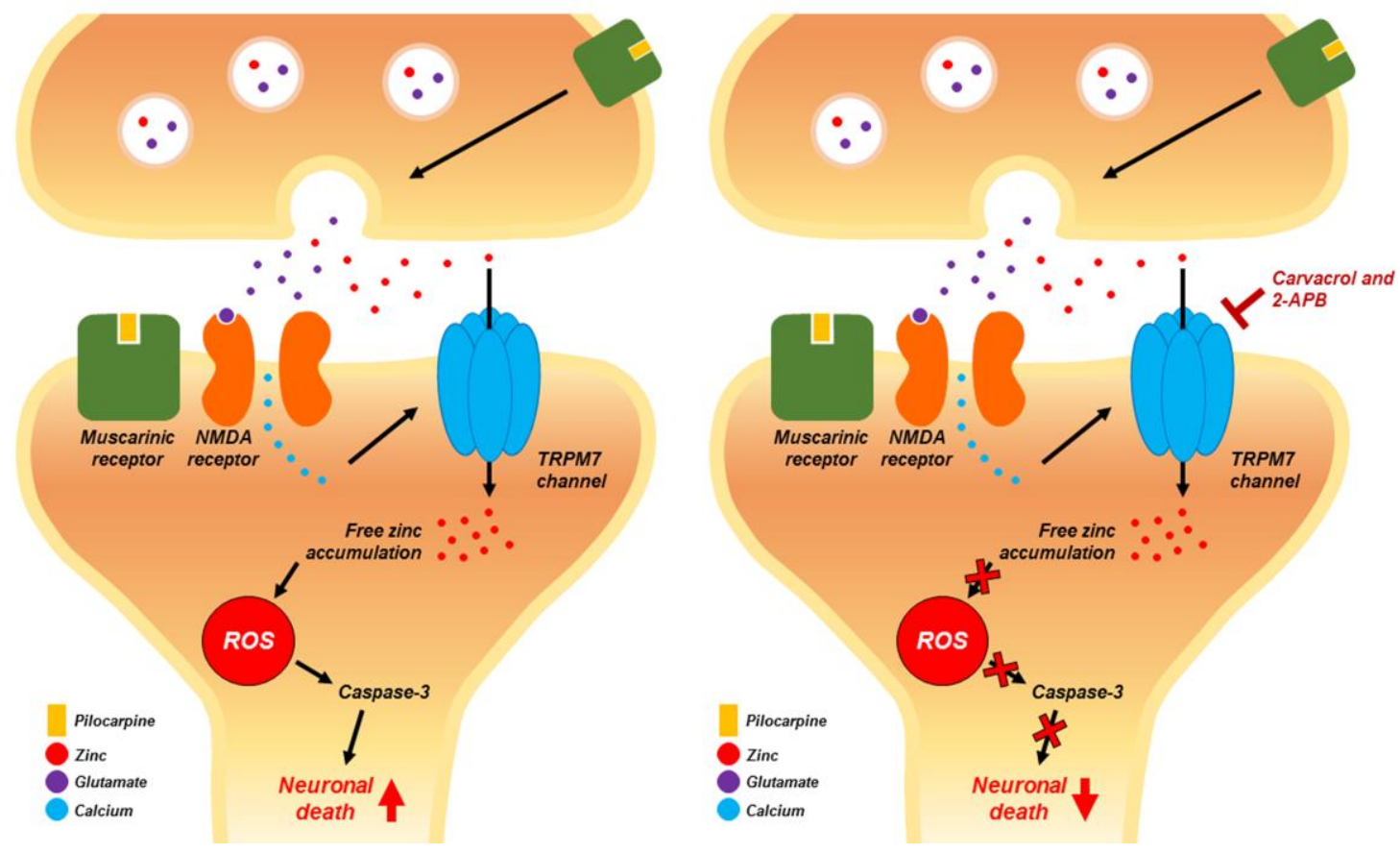

Figure 8. Possible association of zinc with neuronal death after pilocarpine-induced SE. This schematic drawing represents several chain reactions that may occur after carvacrol and 2-APB treatment in pilocarpine-induced SE. (A) These are the possible cellular pathways through which neuronal death occurs after pilocarpine-induced SE. (B) Blocking TRPM7 by carvacrol and 2-APB can inhibit several chain reactions that are thought to occur following pilocarpine-induced SE.

Limitations of this study are that no highly-specific inhibitors of the TRPM7 channel are currently available, and also a paucity of potential mechanisms to explain the neuroprotective effects of the unspecific inhibitors carvacrol and 2-APB on seizure-induced neuronal death. To further confirm our hypothesis a specific TRPM7 inhibitor is needed for future experiments, since both carvacrol and 2-APB are non-specific inhibitors of TRPM7.

Taken together, our findings indicate that TRPM7 inhibition by carvacrol or 2APB can protect seizure-induced neuronal death via the reduction of intracellular free zinc accumulation. 
Thus, the present study suggests that TRPM7 channel inhibition may be a potential candidate for preventing epilepsy-induced neuronal death.

\section{Materials and Methods}

\subsection{Ethics Statement and Experimental Animals}

This study was approved in accordance with the Laboratory Animal Guides and Laboratory Animal Rules published by the National Institute of Health. The animal study was conducted in accordance with the experimental animal research project approved under the criteria of the Experimental Animal Research Committee (Protocol \# Hallym 2018-29). This study was conducted using 8-week-old male Sprague-Dawley rats (280-350 g, DBL Co, Korea). The animals were adapted for 1 week under a constant temperature $\left(22 \pm 2{ }^{\circ} \mathrm{C}\right)$ and humidity $(55 \pm 5 \%)$. Moreover, the room lighting was set to automatically turn on and turn off every $12 \mathrm{~h}$ (8:00 and 20:00) to keep it constant. The study was conducted and written according to the Animal Research: Reporting in Vivo Experiments (ARRIVE) guidelines.

\subsection{Seizure Induction}

Seizures were induced using lithium-pilocarpine. Rats were intraperitoneally injected with lithium chloride $(\mathrm{LiCl}, 127 \mathrm{mg} / \mathrm{kg})$ to boost the action of the muscarinic receptor $19 \mathrm{~h}$ before the injection of pilocarpine. Scopolamine $(2 \mathrm{mg} / \mathrm{kg})$ was intraperitoneally injected $30 \mathrm{~min}$ before pilocarpine injection, which was used to inhibit the peripheral cholinergic properties based on the activity of muscarinic receptors. Pilocarpine $(25 \mathrm{mg} / \mathrm{kg})$ was intraperitoneally injected $30 \mathrm{~min}$ after scopolamine injection. Status epilepticus (SE) was induced following the injection of pilocarpine [105]. All solutions were dissolved with $0.9 \%$ saline and prepared immediately prior to use. SE usually occurs within 20-30 min after pilocarpine injection [106]. The animals were placed one animal per cage to observe their seizure behavior. Seizure behavior was classified according to the process of Racine (facial movements, salivation, hardened hind limbs, forelimb clonus, rearing, and falling) [55]. When Racine stage falling occurs, it is regarded as the onset of seizure. Then, diazepam $(10 \mathrm{mg} / \mathrm{kg})$ was intraperitoneally injected $2 \mathrm{~h}$ after onset to terminate the seizure induction.

\subsection{Experimental Design and Inhibitor Treatment}

To investigate whether carvacrol reduces neuronal death after seizure, the experimental group was divided into the following periods. In phase 1, carvacrol (Sigma-Aldrich Co., St. Louis, MO, USA) was administered once, and the rats were sacrificed $3 \mathrm{~h}$ after seizure. To measure the level of dihydroethidium (dHEt), the animals were divided into 4 groups: (1) sham-vehicle $(n=6),(2)$ sham-carvacrol $(n=6)$, (3) seizure-vehicle $(n=6)$, and (4) seizure-carvacrol $(n=5)$. In phase 2 , carvacrol was administered once, and the rats were sacrificed $24 \mathrm{~h}$ after seizure. To access the level of zinc translocation, the rats were divided into 4 groups: (1) sham-vehicle $(n=6),(2)$ sham-carvacrol $(n=6),(3)$ seizure-vehicle $(n=5)$, and (4) seizure-carvacrol $(n=5)$. In phase 3, carvacrol was administered once a day for 3 days, and the animal brain was obtained 3 days after seizure. To investigate the effect of carvacrol after seizure, the animals were divided into 4 groups: (1) sham-vehicle $(n=5),(2)$ sham-carvacrol $(n=5)$, (3) seizure-vehicle $(n=8)$, and (4) seizure-carvacrol $(n=8)$. In phase 4 , carvacrol was intraperitoneal administered once a day for 7 days, and the experimental animals were sacrificed to obtain brain tissue 7 days after seizure. To evaluate the neuroprotective effect of carvacrol, the experimental animals were divided into 4 groups: (1) sham-vehicle $(n=6),(2)$ sham-carvacrol $(n=6)$, (3) seizure-vehicle $(n=10)$, and (4) seizure-carvacrol $(n=10)$. In phase 5, 2-APB was administered once a day for 3 days, and the brain was obtained at 3 days after seizure. To investigate the effects of 2-APB after seizure, the animals were divided into 4 groups: (1) sham-vehicle $(n=5),(2)$ sham-2PB $(n=5),(3)$ seizure-vehicle $(n=5)$, and (4) seizure-2-APB $(n=6)$. 
Carvacrol was dissolved in $0.1 \%$ DMSO and intraperitoneally administered at a dose of $50 \mathrm{mg} / \mathrm{kg}$ immediately after a 2-h seizure. Control rats were intraperitoneally injected with equal volumes of $0.1 \%$ DMSO (diluted $0.9 \%$ saline) only (vehicle). The concentration of carvacrol was determined in the previous study [47]. The therapeutic window for carvacrol is calculated as LD50/ED50. LD50 is $1544.5 \mathrm{mg} / \mathrm{kg}$, and ED50 is $35.8 \mathrm{mg} / \mathrm{kg}$, so it is 43.142 . Since the dose we used in this experiment is significantly lower than the LD50 concentration. Thus, we believe that the dose we used in this study is sufficiently non-toxic for therapeutic use. In addition, to investigate whether 2-APB reduces neuronal death after seizure, 2-APB was administered once a day for 3 days, and the animal brain was obtained 3 days after seizure. 2-APB was dissolved in $0.9 \%$ saline and intraperitoneally administered at a dose of $2 \mathrm{mg} / \mathrm{kg}$ immediately after a 2-h seizure. Control rats were intraperitoneally injected with equal volumes of $0.9 \%$ saline only (vehicle). The concentration of 2 -APB was determined in the previous study [107].

\subsection{Brain Sample Preparation}

Animals were sacrificed at $3 \mathrm{~h}, 24 \mathrm{~h}, 3$ days, and 7 days after seizure. The animals were anesthetized by an intraperitoneal injection of urethane at a dose of $1.5 \mathrm{~g} / \mathrm{kg}$. After anesthesia, the animals were transcardially perfused with $0.9 \%$ saline and then with $4 \%$ paraformaldehyde. Their brains were quickly removed and post-fixed in $4 \%$ paraformaldehyde for one hour. After fixation, the brains were stored in $30 \%$ sucrose solution, which served as a cryoprotectant. Two days later, the brain was cut to a thickness of $30 \mu \mathrm{m}$ using cryostat microtome (CM1850; Leica, Wetzlar, Germany).

\subsection{Detection of Neuronal Degeneration}

Fluoro-Jade B (FJB) staining was performed to evaluate the effect of carvacrol on neuronal degeneration after seizure. FJB staining was carried out as defined by Schmued et al. [59,108]. After cryosection, the tissue cut to a thickness of $30 \mu \mathrm{m}$ was placed on a slide coated with gelatin. Then, the slide was immersed in 100\% and 70\% alcohol for 3 and $1 \mathrm{~min}$, respectively. The slide was next washed with DW for $1 \mathrm{~min}$ and immersed in $0.06 \%$ potassium permanganate for $15 \mathrm{~min}$. Then, it was washed for $1 \mathrm{~min}$ with DW and immersed in $0.001 \%$ Fluoro-Jade B solution (Histo-Chem Inc., Jefferson, AR, USA) for $30 \mathrm{~min}$ and next washed with DW, dried in an oven for at least $30 \mathrm{~min}$, and the slide was covered with DPX (Sigma-Aldrich Co., St. Louis, MO, USA). The stained tissue was observed at 450-490 nm using an Axioscope microscope (Carl Zeiss, Munchen Hallbergmoos, Germany). Every 6 th section was selected from bregma to caudal from 2.92 to $4.56 \mathrm{~nm}$. The quantification of FJB-positive neurons was conducted by a blind observer.

\subsection{Detection of Zinc Translocation}

6-Methoxy-(8-p-toluene sulfonamido) quinolone (TSQ) staining was performed to evaluate the effect of carvacrol on zinc translocation after seizure. After $24 \mathrm{~h}$ of administration of carvacrol, the rat was anesthetized to obtain brain tissue, and the obtained brain tissue was frozen in dry ice for $1 \mathrm{~min}$. After that, cryosection was carried out with a thickness of $10 \mu \mathrm{m}$. The cut slices were stuck to the coated slides and stained with $0.001 \%$ TSQ solution (Molecular Probes, Eugene, OR, United States) for $1 \mathrm{~min}$. Then, the slides were washed with $0.9 \%$ saline for $1 \mathrm{~min}$ and observed with an Axioscope microscope (Carl Zeiss, Munchen Hallbergmoos, Germany). Quantification of the TSQ positive cells was conducted by a blind observer according to the previously described method [109].

\subsection{Detection of Reactive Oxygen Species (ROS) Activation}

Dihydroethidium (dHEt) staining was performed to evaluate the effects of carvacrol on reactive oxygen species (ROS) after seizure. When rats were injected with pilocarpine, dHEt was also injected $(5 \mathrm{mg} / \mathrm{kg}$, life technologies, Carlsbad, CA, USA). At $3 \mathrm{~h}$ after seizure induction, we obtained brain tissue. The obtained brain tissue was frozen after being sectioned with a thickness of $30 \mu \mathrm{m}$. Then, the tissue was washed three times in phosphate-buffered saline (PBS) for $10 \mathrm{~min}$, placed on a coated slide, 
and mounted with DPX solution. The stained tissue was observed at wavelength of $518 \mathrm{~nm} / 605 \mathrm{~nm}$ using an Axioscope microscope (Carl Zeiss, Munchen Hallbergmoos, Germany). The ethidium signal intensity was quantified by obtaining 5 coronal sections (2.92 to $4.56 \mathrm{~nm}$ from bregma to caudal). Et fluorescence intensity was measured by Image (National Institutes of Health, Bethesda, MD) and expressed as mean gray value.

\subsection{Immunofluorescence Assay}

To evaluate the effect of carvacrol treatment after seizure, we performed an immunofluorescence assay. Brain tissue was obtained 3 days after seizure. The obtained brain tissue was frozen after being sectioned to a thickness of $30 \mu \mathrm{m}$. Then, the tissue was washed three times in PBS for $10 \mathrm{~min}$. Next, pretreatment was performed with a solution containing $90 \%$ methanol, distilled water, and $30 \%$ hydrogen peroxide to completely remove blood from the blood vessels in the tissues. The brain tissue was then immersed in an antibody solution in PBS containing 0.3\% Triton X-100 and kept overnight at $4{ }^{\circ} \mathrm{C}$. The primary antibodies used in this study were as follows: rabbit anti-TRPM7 (diluted 1:400; Alomone labs, Jerusalem, Israel), mouse andti-4HNE (diluted 1:500; Alpha Diagnostic Intl. Inc., San Antonio, TX, USA), goat anti-Iba1 (diluted 1:500; Abcam, Cambridge, UK), mouse anti-CD68 (diluted 1:100; Bio-Rad, Hercules, CA, USA), rabbit anti-GFAP (diluted 1:1k; Abcam, Cambridge, UK), mouse anti-SMI71 (diluted 1:500; Covance, Princeton, NJ, USA), rabbit anti-cleaved caspase3 (diluted 1:250; Cell signaling, Danvers, MA, USA), and mouse anti-NeuN (diluted 1:500; Millipore, Billerica, MA, USA). Next, the tissue was washed three times in PBS for $10 \mathrm{~min}$ and immersed with the secondary antibody in PBS containing $0.3 \%$ Triton X-100 for $2 \mathrm{~h}$. A secondary antibody with fluorescence for each primary antibody (TRPM7, 4HNE, Iba1, CD68, GFAP, SMI71, and cleaved caspase 3) was used (diluted 1:250; Invitrogen, Grand Island, NY, USA). We also counterstained with DAPI (4,6-diamidino-2-phenylindole; diluted 1:1k; Invitrogen, Grand Island, NY, USA). Then, the fluorescence stained sections were placed on a coated slide and mounted with a DPX solution (Sigma-Aldrich). Quantification used the Image J software (National Institutes of Health, Bethesda, MD). This quantification was performed using 5 coronal sections from 2.92 to $4.56 \mathrm{~nm}$ from bregma to caudal. This was quantified using a method modified from the previously described method [110].

\subsection{Immunohistochemistry Assay}

To evaluate the effect of carvacrol treatment after seizure, we performed an immunohistochemistry assay. The brain tissue prepared as above was immersed in a solution of mouse anti-NeuN antibodies (diluted 1:500, Millipore, Billerica, MA, USA) in PBS containing 0.3\% Triton X-100 and kept overnight at $4{ }^{\circ} \mathrm{C}$. Then, the brain tissue was immersed in a solution of anti-mouse IgG (diluted 1:250; Vector, Burlingame, CA, USA) in PBS containing 0.3\% Triton X-100 and treated for $2 \mathrm{~h}$. To analyze endogenous IgG leakage after seizure, sections were incubated in the anti-rat IgG (diluted 1:250; vector, Burlingame, CA, USA) at room temperature for $2 \mathrm{~h}$. Then, the ABC complex solution (Vector, Burlingame, CA, USA) was treated at room temperature for $2 \mathrm{~h}$. The tissue was colored for $1 \mathrm{~min} 30 \mathrm{~s}$ using 3,3'-diaminobenzidine (DAB ager, Sigma-Aldrich Co., St. Louis, Mo, USA) dissolved in a 0.01 M PBS buffer. Then, the stained tissue was placed on a coated slide and mounted in a Canada balsam (Junsei chemical, Chuo-ku, Tokyo, Japan) solution. The quantification used the Image J software (National Institutes of Health, Bethesda, MD) to measure the IgG intensity and NeuN positive cells. The analysis was performed from bregma to caudal with coronal sections obtained from 2.92 to $4.56 \mathrm{~nm}$.

\subsection{Western Blot}

Hippocampal tissues were homogenized with lysis buffer containing RIPA buffer (Cat.IBS-BR002, iNtRON, Seongnam, Republic of Korea), protease inhibitor (Cat.11697498001, Sigma, St. Louis, MO, USA) and phosphatase inhibitor (Cat.4906845001, Sigma, St. Louis, MO, USA). The tissue was incubated on ice for $30 \mathrm{~min}$ and centrifuged at $14,000 \mathrm{rpm}$ for $20 \mathrm{~min}$ at $4{ }^{\circ} \mathrm{C}$. Then, only the 
supernatant was obtained, and protein quantification was carried out using a Bradford protein assay. The quantified protein was prepared at a concentration of $25 \mu \mathrm{g}$ and electrophoresed on SDS-PAGE gel. Then, the separated proteins were transferred to PVDF membrane. The membrane was blocked by incubating 5\% skim milk and 3\% BSA at room temperature to prevent non-specific staining. The membrane was incubated with primary antibody overnight at $4{ }^{\circ} \mathrm{C}$. We used the following primary antibodies: TRPM7 (diluted 1:200; Alomone labs), and $\beta$-actin (diluted 1:10,000; Cell signaling). The membrane was then washed with TBS-T (Cat.190-6435, Bio-Rad, Hercules, CA, USA) three times for $10 \mathrm{~min}$. The membrane was incubated for $1 \mathrm{~h}$ at room temperature using anti-rabbit IgG and anti-mouse IgG secondary antibody conjugated with horseradish peroxidase (HRP) (diluted 1:5000; $\mathrm{Ab}$ frontier). The protein on the PVDF membrane was detected using chemiluminescence bioimaging instrument (Amersham imager 680, Marlborough, USA). The intensities of bands were measured by densitometry using a scanner with Image J (National Institute of Health, Bethesda, MD, USA) and quantitative analysis was peformed.

\subsection{Data Analysis}

In this study, a program called image J (National Institute of Health, Bethesda, MD, USA) was used for data analysis, and the mean gray value or $\%$ area was measured according to the experiment to be analyzed. In order to analyze the number of neurons, the blind observer excluding the experimenter directly counted the number of neurons through the blind test. All data were expressed as the mean \pm SEM. Comparisons between the vehicle- and carvacrol-treated groups were performed with a two-tailed unpaired Student's t-test and a Mann-Whitney $U$ test. To compare the values among the four groups, the remaining data were analyzed by a Kruskal-Wallis test with a post-hoc analysis using Bonferroni correction. $p$-values below 0.05 were considered statistically significant.

Author Contributions: J.H.J. researched the data and wrote the original draft; S.H.L., A.R.K., D.K.H., D.H.K., B.S.K., and M.K.P. researched the data; B.Y.C., H.C.C., M.-S.L. and S.W.S. contributed to the conceptualization, supervision, and discussion and wrote, reviewed, and edited the manuscript. All authors have read and agreed to the published version of the manuscript.

Funding: This study was supported by funding from the National Research Foundation of Korea (NRF) (NRF-2019R1A2C4004912) for Bo Young Choi. This work was also supported by the Brain Research Program through the NRF, funded by the Ministry of Science, Information and Communication Technology and Future Planning (NRF-2017R1D1A1A09081190 and 2020R1A2C2008480) to Sang Won Suh.

Acknowledgments: This research was supported by Hallym University Research Fund.

Conflicts of Interest: The authors declare no conflict of interest.

\section{References}

1. Thurman, D.J.; Beghi, E.; Begley, C.E.; Berg, A.T.; Buchhalter, J.R.; Ding, D.; Hesdorffer, D.C.; Hauser, W.A.; Kazis, L.; Kobau, R.; et al. Standards for epidemiologic studies and surveillance of epilepsy. Epilepsia 2011, 52 (Suppl. S7), 2-26. [CrossRef]

2. Fisher, R.S.; Acevedo, C.; Arzimanoglou, A.; Bogacz, A.; Cross, J.H.; Elger, C.E.; Engel, J., Jr.; Forsgren, L.; French, J.A.; Glynn, M.; et al. ILAE official report: A practical clinical definition of epilepsy. Epilepsia 2014, 55, 475-482. [CrossRef] [PubMed]

3. Chang, Y.C.; Huang, A.M.; Kuo, Y.M.; Wang, S.T.; Chang, Y.Y.; Huang, C.C. Febrile seizures impair memory and cAMP response-element binding protein activation. Ann. Neurol. 2003, 54, 706-718. [CrossRef] [PubMed]

4. Holmes, G.L.; Gairsa, J.L.; Chevassus-Au-Louis, N.; Ben-Ari, Y. Consequences of neonatal seizures in the rat: Morphological and behavioral effects. Ann. Neurol. 1998, 44, 845-857. [CrossRef] [PubMed]

5. Delgado-Escueta, A.V.; Wilson, W.A.; Olsen, R.W.; Porter, R.J. New waves of research in the epilepsies: Crossing into the third millennium. Adv. Neurol. 1999, 79, 3-58.

6. Quinlan, S.M.M.; Rodriguez-Alvarez, N.; Molloy, E.J.; Madden, S.F.; Boylan, G.B.; Henshall, D.C.; Jimenez-Mateos, E.M. Complex spectrum of phenobarbital effects in a mouse model of neonatal hypoxia-induced seizures. Sci. Rep. 2018, 8, 9986. [CrossRef] 
7. Kofuji, P.; Newman, E.A. Potassium buffering in the central nervous system. Neuroscience 2004, 129, 1045-1056. [CrossRef]

8. Stafstrom, C.E. Persistent sodium current and its role in epilepsy. Epilepsy Curr. 2007, 7, 15-22. [CrossRef]

9. Bozzi, Y.; Provenzano, G.; Casarosa, S. Neurobiological bases of autism-epilepsy comorbidity: A focus on excitation/inhibition imbalance. Eur. J. Neurosci. 2018, 47, 534-548. [CrossRef]

10. Griffiths, T.; Evans, M.C.; Meldrum, B.S. Intracellular calcium accumulation in rat hippocampus during seizures induced by bicuculline or L-allylglycine. Neuroscience 1983, 10, 385-395. [CrossRef]

11. Al-Mufti, F.; Claassen, J. Neurocritical care: Status epilepticus review. Crit. Care Clin. 2014, 30, 751-764. [CrossRef] [PubMed]

12. Vingerhoets, G. Cognitive effects of seizures. Seizure 2006, 15, 221-226. [CrossRef] [PubMed]

13. Ryvlin, P.; Kahane, P. Does epilepsy surgery lower the mortality of drug-resistant epilepsy? Epilepsy Res. 2003, 56, 105-120. [CrossRef] [PubMed]

14. Bertram, E.H. Temporal lobe epilepsy: Where do the seizures really begin? Epilepsy Behav. 2009, 14 (Suppl. S1), 32-37. [CrossRef]

15. Portbury, S.D.; Adlard, P.A. Zinc Signal in Brain Diseases. Int. J. Mol. Sci. 2017, 18, 2506. [CrossRef]

16. Szewczyk, B. Zinc homeostasis and neurodegenerative disorders. Front. Aging Neurosci. 2013, 5, 33. [CrossRef]

17. MacDonald, R.S. The role of zinc in growth and cell proliferation. J. Nutr. 2000, 130 (Suppl. 5S), 1500S-1508S. [CrossRef]

18. Beyersmann, D.; Haase, H. Functions of zinc in signaling, proliferation and differentiation of mammalian cells. Biometals 2001, 14, 331-341. [CrossRef]

19. Frederickson, C.J.; Hernandez, M.D.; McGinty, J.F. Translocation of zinc may contribute to seizure-induced death of neurons. Brain Res. 1989, 480, 317-321. [CrossRef]

20. Howell, G.A.; Welch, M.G.; Frederickson, C.J. Stimulation-induced uptake and release of zinc in hippocampal slices. Nature 1984, 308, 736-738. [CrossRef]

21. Perez-Clausell, J.; Danscher, G. Intravesicular localization of zinc in rat telencephalic boutons. A histochemical study. Brain Res. 1985, 337, 91-98. [CrossRef]

22. Assaf, S.Y.; Chung, S.H. Release of endogenous Zn2+ from brain tissue during activity. Nature 1984, 308, 734-736. [CrossRef] [PubMed]

23. Suh, S.W.; Jo, S.M.; Vajda, Z.; Danscher, G. Adrenalectomy causes loss of zinc ions in zinc-enriched (ZEN) terminals and decreases seizure-induced neuronal death. Brain Res. 2001, 895, 25-32. [CrossRef]

24. Cote, A.; Chiasson, M.; Peralta, M.R., 3rd; Lafortune, K.; Pellegrini, L.; Toth, K. Cell type-specific action of seizure-induced intracellular zinc accumulation in the rat hippocampus. J. Physiol. 2005, 566, 821-837. [CrossRef]

25. Kim, J.H.; Jang, B.G.; Choi, B.Y.; Kwon, L.M.; Sohn, M.; Song, H.K.; Suh, S.W. Zinc chelation reduces hippocampal neurogenesis after pilocarpine-induced seizure. PLoS ONE 2012, 7, e48543. [CrossRef]

26. Doboszewska, U.; Mlyniec, K.; Wlaz, A.; Poleszak, E.; Nowak, G.; Wlaz, P. Zinc signaling and epilepsy. Pharmacol. Ther. 2019, 193, 156-177. [CrossRef] [PubMed]

27. Jang, B.G.; Won, S.J.; Kim, J.H.; Choi, B.Y.; Lee, M.W.; Sohn, M.; Song, H.K.; Suh, S.W. EAAC1 gene deletion alters zinc homeostasis and enhances cortical neuronal injury after transient cerebral ischemia in mice. J. Trace Elem. Med. Biol. 2012, 26, 85-88. [CrossRef]

28. Choi, B.Y.; Kim, J.H.; Kim, H.J.; Lee, B.E.; Kim, I.Y.; Sohn, M.; Suh, S.W. Zinc chelation reduces traumatic brain injury-induced neurogenesis in the subgranular zone of the hippocampal dentate gyrus. J. Trace Elem. Med. Biol. 2014, 28, 474-481. [CrossRef]

29. Suh, S.W.; Hamby, A.M.; Gum, E.T.; Shin, B.S.; Won, S.J.; Sheline, C.T.; Chan, P.H.; Swanson, R.A. Sequential release of nitric oxide, zinc, and superoxide in hypoglycemic neuronal death. J. Cereb. Blood Flow Metab. 2008, 28, 1697-1706. [CrossRef]

30. Suh, S.W.; Garnier, P.; Aoyama, K.; Chen, Y.; Swanson, R.A. Zinc release contributes to hypoglycemia-induced neuronal death. Neurobiol. Dis. 2004, 16, 538-545.

31. Li, H. TRP Channel Classification. Adv. Exp. Med. Biol. 2017, 976, 1-8. [PubMed]

32. Park, H.S.; Hong, C.; Kim, B.J.; So, I. The Pathophysiologic Roles of TRPM7 Channel. Korean J. Physiol. Pharmacol. 2014, 18, 15-23. [CrossRef] [PubMed] 
33. Monteilh-Zoller, M.K.; Hermosura, M.C.; Nadler, M.J.; Scharenberg, A.M.; Penner, R.; Fleig, A. TRPM7 provides an ion channel mechanism for cellular entry of trace metal ions. J. Gen. Physiol. 2003, 121, 49-60. [CrossRef]

34. Clapham, D.E. TRP channels as cellular sensors. Nature 2003, 426, 517-524. [CrossRef] [PubMed]

35. Inoue, K.; Branigan, D.; Xiong, Z.G. Zinc-induced neurotoxicity mediated by transient receptor potential melastatin 7 channels. J. Biol. Chem. 2010, 285, 7430-7439. [CrossRef] [PubMed]

36. De Vincenzi, M.; Stammati, A.; De Vincenzi, A.; Silano, M. Constituents of aromatic plants: Carvacrol. Fitoterapia 2004, 75, 801-804. [CrossRef]

37. Aligiannis, N.; Kalpoutzakis, E.; Mitaku, S.; Chinou, I.B. Composition and antimicrobial activity of the essential oils of two Origanum species. J. Agric. Food Chem. 2001, 49, 4168-4170. [CrossRef]

38. Dantas, B.P.; Alves, Q.L.; de Assis, K.S.; Ribeiro, T.P.; de Almeida, M.M.; de Vasconcelos, A.P.; de Araujo, D.A.; de Andrade Braga, V.; de Medeiros, I.A.; Alencar, J.L.; et al. Participation of the TRP channel in the cardiovascular effects induced by carvacrol in normotensive rat. Vascul. Pharmacol. 2015, 67-69, 48-58. [CrossRef]

39. Almanaityte, M.; Jurevicius, J.; Macianskiene, R. Effect of Carvacrol, TRP Channels Modulator, on Cardiac Electrical Activity. Biomed. Res. Int. 2020, 2020, 6456805. [CrossRef]

40. Khalil, A.; Kovac, S.; Morris, G.; Walker, M.C. Carvacrol after status epilepticus (SE) prevents recurrent SE, early seizures, cell death, and cognitive decline. Epilepsia 2017, 58, 263-273. [CrossRef]

41. Somensi, N.; Rabelo, T.K.; Guimaraes, A.G.; Quintans-Junior, L.J.; de Souza Araujo, A.A.; Moreira, J.C.F.; Gelain, D.P. Carvacrol suppresses LPS-induced pro-inflammatory activation in RAW 264.7 macrophages through ERK1/2 and NF-kB pathway. Int. Immunopharmacol. 2019, 75, 105743. [CrossRef] [PubMed]

42. Dati, L.M.; Ulrich, H.; Real, C.C.; Feng, Z.P.; Sun, H.S.; Britto, L.R. Carvacrol promotes neuroprotection in the mouse hemiparkinsonian model. Neuroscience 2017, 356, 176-181. [CrossRef] [PubMed]

43. Skalicka-Wozniak, K.; Walasek, M.; Aljarba, T.M.; Stapleton, P.; Gibbons, S.; Xiao, J.; Luszczki, J.J. The anticonvulsant and anti-plasmid conjugation potential of Thymus vulgaris chemistry: An in vivo murine and in vitro study. Food Chem. Toxicol. 2018, 120, 472-478. [CrossRef]

44. Mishra, R.K.; Baker, M.T. Seizure prevention by the naturally occurring phenols, carvacrol and thymol in a partial seizure-psychomotor model. Bioorg. Med. Chem. Lett. 2014, 24, 5446-5449. [CrossRef]

45. Sun, H.S.; Jackson, M.F.; Martin, L.J.; Jansen, K.; Teves, L.; Cui, H.; Kiyonaka, S.; Mori, Y.; Jones, M.; Forder, J.P.; et al. Suppression of hippocampal TRPM7 protein prevents delayed neuronal death in brain ischemia. Nat. Neurosci. 2009, 12, 1300-1307. [CrossRef]

46. Li, W.T.; Zhang, S.Y.; Zhou, Y.F.; Zhang, B.F.; Liang, Z.Q.; Liu, Y.H.; Wei, Y.; Li, C.K.; Meng, X.J.; Xia, M.; et al. Carvacrol attenuates traumatic neuronal injury through store-operated $\mathrm{Ca}(2+)$ entry-independent regulation of intracellular $\mathrm{Ca}(2+)$ homeostasis. Neurochem. Int. 2015, 90, 107-113. [CrossRef]

47. Hong, D.K.; Choi, B.Y.; Kho, A.R.; Lee, S.H.; Jeong, J.H.; Kang, B.S.; Kang, D.H.; Park, K.H.; Suh, S.W. Carvacrol Attenuates Hippocampal Neuronal Death after Global Cerebral Ischemia via Inhibition of Transient Receptor Potential Melastatin 7. Cells 2018, 7, 231. [CrossRef]

48. Hamzehloei, L.; Rezvani, M.E.; Rajaei, Z. Effects of carvacrol and physical exercise on motor and memory impairments associated with Parkinson's disease. Arq. Neuropsiquiatr. 2019, 77, 493-500. [CrossRef]

49. Liu, H.; Dilger, J.P.; Lin, J. The Role of Transient Receptor Potential Melastatin 7 (TRPM7) in Cell Viability: A Potential Target to Suppress Breast Cancer Cell Cycle. Cancers (Basel) 2020, 12, 131. [CrossRef]

50. Bilmen, J.G.; Michelangeli, F. Inhibition of the type 1 inositol 1,4,5-trisphosphate receptor by 2-aminoethoxydiphenylborate. Cell. Signal. 2002, 14, 955-960. [CrossRef]

51. Ma, H.T.; Patterson, R.L.; van Rossum, D.B.; Birnbaumer, L.; Mikoshiba, K.; Gill, D.L. Requirement of the inositol trisphosphate receptor for activation of store-operated Ca2+ channels. Science 2000, 287, 1647-1651. [CrossRef] [PubMed]

52. Ma, H.T.; Venkatachalam, K.; Li, H.S.; Montell, C.; Kurosaki, T.; Patterson, R.L.; Gill, D.L. Assessment of the role of the inositol 1,4,5-trisphosphate receptor in the activation of transient receptor potential channels and store-operated Ca2+ entry channels. J. Biol. Chem. 2001, 276, 18888-18896. [CrossRef] [PubMed]

53. Chokshi, R.; Fruasaha, P.; Kozak, J.A. 2-aminoethyl diphenyl borinate (2-APB) inhibits TRPM7 channels through an intracellular acidification mechanism. Channels (Austin) 2012, 6, 362-369. [CrossRef] [PubMed] 
54. Lipski, J.; Park, T.I.; Li, D.; Lee, S.C.; Trevarton, A.J.; Chung, K.K.; Freestone, P.S.; Bai, J.Z. Involvement of TRP-like channels in the acute ischemic response of hippocampal CA1 neurons in brain slices. Brain Res. 2006, 1077, 187-199. [CrossRef] [PubMed]

55. Racine, R.J.; Gartner, J.G.; Burnham, W.M. Epileptiform activity and neural plasticity in limbic structures. Brain Res. 1972, 47, 262-268. [CrossRef]

56. Meeusen, J.W.; Tomasiewicz, H.; Nowakowski, A.; Petering, D.H. TSQ (6-methoxy-8-p-toluenesulfonamidoquinoline), a common fluorescent sensor for cellular zinc, images zinc proteins. Inorg. Chem. 2011, 50, 7563-7573. [CrossRef]

57. Choi, B.Y.; Lee, B.E.; Kim, J.H.; Kim, H.J.; Sohn, M.; Song, H.K.; Chung, T.N.; Suh, S.W. Colchicine induced intraneuronal free zinc accumulation and dentate granule cell degeneration. Metallomics 2014, 6, 1513-1520. [CrossRef]

58. Choi, B.Y.; Hong, D.K.; Suh, S.W. ZnT3 Gene Deletion Reduces Colchicine-Induced Dentate Granule Cell Degeneration. Int. J. Mol. Sci. 2017, 18, 2189. [CrossRef]

59. Schmued, L.C.; Hopkins, K.J. Fluoro-Jade B: A high affinity fluorescent marker for the localization of neuronal degeneration. Brain Res. 2000, 874, 123-130. [CrossRef]

60. Bolos, M.; Perea, J.R.; Avila, J. Alzheimer's disease as an inflammatory disease. Biomol. Concepts 2017, 8, 37-43. [CrossRef]

61. Mocchegiani, E.; Malavolta, M.; Muti, E.; Costarelli, L.; Cipriano, C.; Piacenza, F.; Tesei, S.; Giacconi, R.; Lattanzio, F. Zinc, metallothioneins and longevity: Interrelationships with niacin and selenium. Curr. Pharm. Des. 2008, 14, 2719-2732. [CrossRef]

62. Kasarskis, E.J.; Forrester, T.M.; Slevin, J.T. Amygdalar kindling is associated with elevated zinc concentration in the cortex and hippocampus of rats. Epilepsy Res. 1987, 1, 227-233. [CrossRef]

63. Yee, N.S.; Kazi, A.A.; Yee, R.K. Cellular and Developmental Biology of TRPM7 Channel-Kinase: Implicated Roles in Cancer. Cells 2014, 3, 751-777. [CrossRef] [PubMed]

64. Kuenssberg, E.V. Reorganisation: Integration or disintegration. Nurs. Times 1974, 70, 458-459.

65. Sun, Y.; Sukumaran, P.; Schaar, A.; Singh, B.B. TRPM7 and its role in neurodegenerative diseases. Channels (Austin) 2015, 9, 253-261. [CrossRef] [PubMed]

66. Aarts, M.; Iihara, K.; Wei, W.L.; Xiong, Z.G.; Arundine, M.; Cerwinski, W.; MacDonald, J.F.; Tymianski, M. A key role for TRPM7 channels in anoxic neuronal death. Cell 2003, 115, 863-877. [CrossRef]

67. Mittmeyer, H.J. [Distribution pattern of rigor mortis in various joint regions]. Beitr. Gerichtl. Med. 1975, 33, 85-96. [PubMed]

68. Penkowa, M.; Molinero, A.; Carrasco, J.; Hidalgo, J. Interleukin-6 deficiency reduces the brain inflammatory response and increases oxidative stress and neurodegeneration after kainic acid-induced seizures. Neuroscience 2001, 102, 805-818. [CrossRef]

69. Swissa, E.; Serlin, Y.; Vazana, U.; Prager, O.; Friedman, A. Blood-brain barrier dysfunction in status epileptics: Mechanisms and role in epileptogenesis. Epilepsy Behav. 2019, 101, 106285. [CrossRef]

70. Eyo, U.B.; Murugan, M.; Wu, L.J. Microglia-Neuron Communication in Epilepsy. Glia 2017, 65, 5-18. [CrossRef]

71. Schieber, M.; Chandel, N.S. ROS function in redox signaling and oxidative stress. Curr. Biol. 2014, 24, R453-R462. [CrossRef]

72. Morrow, G.; Le Pecheur, M.; Tanguay, R.M. Drosophila melanogaster mitochondrial Hsp22: A role in resistance to oxidative stress, aging and the mitochondrial unfolding protein response. Biogerontology 2016, 17, 61-70. [CrossRef]

73. Esmekaya, M.A.; Tuysuz, M.Z.; Tomruk, A.; Canseven, A.G.; Yucel, E.; Aktuna, Z.; Keskil, S.; Seyhan, N. Effects of cell phone radiation on lipid peroxidation, glutathione and nitric oxide levels in mouse brain during epileptic seizure. J. Chem. Neuroanat. 2016, 75, 111-115. [CrossRef] [PubMed]

74. Abiria, S.A.; Krapivinsky, G.; Sah, R.; Santa-Cruz, A.G.; Chaudhuri, D.; Zhang, J.; Adstamongkonkul, P.; DeCaen, P.G.; Clapham, D.E. TRPM7 senses oxidative stress to release $\mathrm{Zn}(2+)$ from unique intracellular vesicles. Proc. Natl. Acad. Sci. USA 2017, 114, E6079-E6088. [CrossRef]

75. Zhao, Y.; Yan, F.; Yin, J.; Pan, R.; Shi, W.; Qi, Z.; Fang, Y.; Huang, Y.; Li, S.; Luo, Y.; et al. Synergistic Interaction Between Zinc and Reactive Oxygen Species Amplifies Ischemic Brain Injury in Rats. Stroke 2018, 49, 2200-2210. [CrossRef] 
76. Morris, G.P.; Clark, I.A.; Zinn, R.; Vissel, B. Microglia: A new frontier for synaptic plasticity, learning and memory, and neurodegenerative disease research. Neurobiol. Learn Mem. 2013, 105, 40-53. [CrossRef]

77. De Simoni, M.G.; Perego, C.; Ravizza, T.; Moneta, D.; Conti, M.; Marchesi, F.; De Luigi, A.; Garattini, S.; Vezzani, A. Inflammatory cytokines and related genes are induced in the rat hippocampus by limbic status epilepticus. Eur. J. Neurosci. 2000, 12, 2623-2633. [CrossRef]

78. Plata-Salaman, C.R.; Ilyin, S.E.; Turrin, N.P.; Gayle, D.; Flynn, M.C.; Romanovitch, A.E.; Kelly, M.E.; Bureau, Y.; Anisman, H.; McIntyre, D.C. Kindling modulates the IL-1beta system, TNF-alpha, TGF-beta1, and neuropeptide mRNAs in specific brain regions. Brain Res. Mol. Brain Res. 2000, 75, 248-258. [CrossRef]

79. Rizzi, M.; Perego, C.; Aliprandi, M.; Richichi, C.; Ravizza, T.; Colella, D.; Veliskova, J.; Moshe, S.L.; De Simoni, M.G.; Vezzani, A. Glia activation and cytokine increase in rat hippocampus by kainic acid-induced status epilepticus during postnatal development. Neurobiol. Dis. 2003, 14, 494-503. [CrossRef]

80. Subhramanyam, C.S.; Wang, C.; Hu, Q.; Dheen, S.T. Microglia-mediated neuroinflammation in neurodegenerative diseases. Semin. Cell Dev. Biol. 2019, 94, 112-120. [CrossRef]

81. Magni, P.; Ruscica, M.; Dozio, E.; Rizzi, E.; Beretta, G.; Maffei Facino, R. Parthenolide inhibits the LPS-induced secretion of IL-6 and TNF-alpha and NF-kappaB nuclear translocation in BV-2 microglia. Phytother. Res. 2012, 26, 1405-1409. [CrossRef] [PubMed]

82. Benson, M.J.; Manzanero, S.; Borges, K. Complex alterations in microglial M1/M2 markers during the development of epilepsy in two mouse models. Epilepsia 2015, 56, 895-905. [CrossRef] [PubMed]

83. Xu, N.; Tang, X.H.; Pan, W.; Xie, Z.M.; Zhang, G.F.; Ji, M.H.; Yang, J.J.; Zhou, M.T.; Zhou, Z.Q. Spared Nerve Injury Increases the Expression of Microglia M1 Markers in the Prefrontal Cortex of Rats and Provokes Depression-Like Behaviors. Front. Neurosci. 2017, 11, 209. [CrossRef] [PubMed]

84. Serlin, Y.; Shelef, I.; Knyazer, B.; Friedman, A. Anatomy and physiology of the blood-brain barrier. Semin. Cell Dev. Biol. 2015, 38, 2-6. [CrossRef]

85. Neuwelt, E.A.; Bauer, B.; Fahlke, C.; Fricker, G.; Iadecola, C.; Janigro, D.; Leybaert, L.; Molnar, Z.; O'Donnell, M.E.; Povlishock, J.T.; et al. Engaging neuroscience to advance translational research in brain barrier biology. Nat. Rev. Neurosci. 2011, 12, 169-182. [CrossRef]

86. Mendes, N.F.; Pansani, A.P.; Carmanhaes, E.R.F.; Tange, P.; Meireles, J.V.; Ochikubo, M.; Chagas, J.R.; da Silva, A.V.; Monteiro de Castro, G.; Le Sueur-Maluf, L. The Blood-Brain Barrier Breakdown During Acute Phase of the Pilocarpine Model of Epilepsy Is Dynamic and Time-Dependent. Front. Neurol. 2019, 10, 382. [CrossRef]

87. Cornford, E.M.; Oldendorf, W.H. Epilepsy and the blood-brain barrier. Adv. Neurol. 1986, 44, 787-812.

88. Cornford, E.M. Epilepsy and the blood brain barrier: Endothelial cell responses to seizures. Adv. Neurol. 1999, 79, 845-862.

89. Obermeier, B.; Daneman, R.; Ransohoff, R.M. Development, maintenance and disruption of the blood-brain barrier. Nat. Med. 2013, 19, 1584-1596. [CrossRef]

90. Prager, O.; Kamintsky, L.; Hasam-Henderson, L.A.; Schoknecht, K.; Wuntke, V.; Papageorgiou, I.; Swolinsky, J.; Muoio, V.; Bar-Klein, G.; Vazana, U.; et al. Seizure-induced microvascular injury is associated with impaired neurovascular coupling and blood-brain barrier dysfunction. Epilepsia 2019, 60, 322-336. [CrossRef] [PubMed]

91. Friedman, A. Blood-brain barrier dysfunction, status epilepticus, seizures, and epilepsy: A puzzle of a chicken and egg? Epilepsia 2011, 52 (Suppl. S8), 19-20. [CrossRef] [PubMed]

92. Correale, J.; Rabinowicz, A.L.; Heck, C.N.; Smith, T.D.; Loskota, W.J.; DeGiorgio, C.M. Status epilepticus increases CSF levels of neuron-specific enolase and alters the blood-brain barrier. Neurology 1998, 50, 1388-1391. [CrossRef] [PubMed]

93. Jung, H.K.; Ryu, H.J.; Kim, M.J.; Kim, W.I.; Choi, H.K.; Choi, H.C.; Song, H.K.; Jo, S.M.; Kang, T.C. Interleukin-18 attenuates disruption of brain-blood barrier induced by status epilepticus within the rat piriform cortex in interferon-gamma independent pathway. Brain Res. 2012, 1447, 126-134. [CrossRef] [PubMed]

94. Zengin Kurt, B.; Durdagi, S.; Celebi, G.; Ekhteiari Salmas, R.; Sonmez, F. Synthesis, anticholinesterase activity and molecular modeling studies of novel carvacrol-substituted amide derivatives. J. Biomol. Struct. Dyn. 2020, 38, 841-859. [CrossRef] 
95. Trabace, L.; Zotti, M.; Morgese, M.G.; Tucci, P.; Colaianna, M.; Schiavone, S.; Avato, P.; Cuomo, V. Estrous cycle affects the neurochemical and neurobehavioral profile of carvacrol-treated female rats. Toxicol. Appl. Pharmacol. 2011, 255, 169-175. [CrossRef]

96. Nostro, A.; Papalia, T. Antimicrobial activity of carvacrol: Current progress and future prospectives. Recent. Pat. Antiinfect. Drug Discov. 2012, 7, 28-35. [CrossRef]

97. de Castro, R.D.; de Souza, T.M.; Bezerra, L.M.; Ferreira, G.L.; Costa, E.M.; Cavalcanti, A.L. Antifungal activity and mode of action of thymol and its synergism with nystatin against Candida species involved with infections in the oral cavity: An in vitro study. BMC Complement Altern. Med. 2015, 15, 417. [CrossRef]

98. Millan, M.H.; Chapman, A.G.; Meldrum, B.S. Extracellular amino acid levels in hippocampus during pilocarpine-induced seizures. Epilepsy Res. 1993, 14, 139-148. [CrossRef]

99. MacDonald, J.F.; Xiong, Z.G.; Jackson, M.F. Paradox of Ca2+ signaling, cell death and stroke. Trends Neurosci. 2006, 29, 75-81. [CrossRef]

100. Bae, C.Y.; Sun, H.S. TRPM7 in cerebral ischemia and potential target for drug development in stroke. Acta Pharmacol. Sin. 2011, 32, 725-733. [CrossRef]

101. May, J.M.; Contoreggi, C.S. The mechanism of the insulin-like effects of ionic zinc. J. Biol. Chem. 1982, 257, 4362-4368. [PubMed]

102. Bishop, G.M.; Dringen, R.; Robinson, S.R. Zinc stimulates the production of toxic reactive oxygen species (ROS) and inhibits glutathione reductase in astrocytes. Free Radic. Biol. Med. 2007, 42, 1222-1230. [CrossRef]

103. Beckman, J.S.; Crow, J.P. Pathological implications of nitric oxide, superoxide and peroxynitrite formation. Biochem. Soc. Trans. 1993, 21, 330-334. [CrossRef]

104. Kim, Y.H.; Kim, E.Y.; Gwag, B.J.; Sohn, S.; Koh, J.Y. Zinc-induced cortical neuronal death with features of apoptosis and necrosis: Mediation by free radicals. Neuroscience 1999, 89, 175-182. [CrossRef]

105. Van Rijckevorsel, K. Cognitive problems related to epilepsy syndromes, especially malignant epilepsies. Seizure 2006, 15, 227-234. [CrossRef] [PubMed]

106. Persinger, M.A.; Makarec, K.; Bradley, J.C. Characteristics of limbic seizures evoked by peripheral injections of lithium and pilocarpine. Physiol. Behav. 1988, 44, 27-37. [CrossRef]

107. Boyaci, M.G.; Rakip, U.; Aslan, A.; Koca, H.B.; Aslan, E.; Korkmaz, S.; Yildizhan, S. Effects of 2-Aminoethyl Diphenylborinate, a Modulator of Transient Receptor Potential and Orai Channels in Subarachnoid Hemorrhage: An Experimental Study. World Neurosurg. 2019, 127, e376-e388. [CrossRef]

108. Suh, S.W.; Aoyama, K.; Chen, Y.; Garnier, P.; Matsumori, Y.; Gum, E.; Liu, J.; Swanson, R.A. Hypoglycemic neuronal death and cognitive impairment are prevented by poly(ADP-ribose) polymerase inhibitors administered after hypoglycemia. J. Neurosci. 2003, 23, 10681-10690. [CrossRef]

109. Frederickson, C.J.; Kasarskis, E.J.; Ringo, D.; Frederickson, R.E. A quinoline fluorescence method for visualizing and assaying the histochemically reactive zinc (bouton zinc) in the brain. J. Neurosci. Methods 1987, 20, 91-103. [CrossRef]

110. Tang, X.N.; Berman, A.E.; Swanson, R.A.; Yenari, M.A. Digitally quantifying cerebral hemorrhage using Photoshop and Image J. J. Neurosci. Methods 2010, 190, 240-243. [CrossRef]

Publisher's Note: MDPI stays neutral with regard to jurisdictional claims in published maps and institutional affiliations.

(C) 2020 by the authors. Licensee MDPI, Basel, Switzerland. This article is an open access article distributed under the terms and conditions of the Creative Commons Attribution (CC BY) license (http://creativecommons.org/licenses/by/4.0/). 